\title{
Identification of a ferroptosis-related IncRNA signature with prognosis for Wilms tumor
}

\author{
Hengchen Liu", Mingzhao Zhang”, Tingting Zhang", Manyu Shi, Wenjun Lu, Shulong Yang, Qingbo Cui, \\ Zhaozhu Li^
}

Department of Pediatric Surgery, The Second Hospital Affiliated to Harbin Medical University, Harbin, China

Contributions: (I) Conception and design: H Liu, Z Li; (II) Administrative support: Z Li, Q Cui; (III) Provision of study materials or patients: H Liu, M Zhang, T Zhang; (IV) Collection and assembly of data: M Shi, W Lu; (V) Data analysis and interpretation: H Liu, S Yang; (VI) Manuscript writing: All authors; (VII) Final approval of manuscript: All authors.

\#These authors contributed equally to this work.

Correspondence to: Zhaozhu Li. Department of Pediatric Surgery, The Second Affiliated Hospital of Harbin Medical University, No. 246, Xuefu Road, Nangang District, Harbin 150001, China. Email: zhaozhu247@163.com; Qingbo Cui. Department of Pediatric Surgery, The Second Affiliated Hospital of Harbin Medical University, No. 246, Xuefu Road, Nangang District, Harbin 150001, China. Email: cuiqingbocqb@163.com.

Background: Wilms tumor (WT) is a widespread urologic tumor in children. Ferroptosis, on the other hand, is a novel form of cell death associated with tumor development. In this study, we aim to explore the predictability of ferroptosis-related biomarkers in estimating prognosis in WT patients.

Methods: To determine a link between ferroptosis-related gene expression and WT prognosis, we first collected RNA sequencing data and clinical information, involving $124 \mathrm{WT}$ and 6 healthy tissue samples, from the TARGET database. Next, we screened the collected information for ferroptosis-related long noncoding RNA using Cox regression analysis, and constructed a signature model, as well as a nomogram, related to prognosis. Finally, we explored a potential link between ferroptosis-related lncRNA and tumor immunity and screened for possible immune checkpoints.

Results: We constructed a WT prognosis prediction signature containing 12 ferroptosis-related lncRNAs. The area under the curves values, from the ROC curves, predicting overall survival rates at the 1, 3-, and 5 -year timepoints were $0.775,0.867$, and 0.891 respectively. Moreover, we generated a nomogram, using clinical features and risk scores, carrying a C-index value of 0.836 , which suggested a high predictive value. We also demonstrated significant differences in tumor immunity between low- and high-risk WT patients, particularly in the presence of B cells, NK cells, Th1 cells, Treg cells, inflammation promoting, and type I and II IFN responses. In addition, we showed that immune checkpoints like SIRPA, ICOSLG, LAG3, PVRIG, NECTIN1, and SIRPB2 can serve as potential therapeutic targets for W'T.

Conclusions: Based on our analyses, we generated a ferroptosis-related lncRNA signature that can both estimate prognosis of WT patients and may provide basis for future WT therapy.

Keywords: Wilms tumor (WT); TARGET; ferroptosis; lncRNAs; prognosis

Submitted May 11, 2021. Accepted for publication Aug 05, 2021.

doi: $10.21037 / \mathrm{tp}-21-211$

View this article at: https://dx.doi.org/10.21037/tp-21-211

^ ORCID: 0000-0002-8953-8186. 


\section{Introduction}

Wilms tumor (WT) is a common embryonal kidney tumor that is prevalent among children $<5$ years of age (1). Being the fifth ranking childhood malignancy in the world, 1 in 10,000 children typically experience abdominal mass or hematuria and are diagnosed with WT (2). In the 1930s, the survival rate of WT patients was $<30 \%$. Fortunately, with advancements in medical care, radical resection, and chemoradiotherapy, the survival rate has improved to $>90 \%$ (3). However, the high-intensity chemoradiotherapy produce side effects that severely affect quality of life in WT patients $(4,5)$. Meanwhile, there are no other treatment options for patients with poor tumor stage, bilateral lesions, and recurrence $(6,7)$. Therefore, clarifying WT molecular mechanism, identifying potential therapeutic targets, and developing individualized treatment for WT are keys to improving patient prognosis and survival.

In recent years, ferroptosis has been widely regarded as a novel type of regulated cell death (8). The concept of ferroptosis was first proposed by Dixon et al. in 2012 (9). Unlike traditional cell necrosis and apoptosis, ferroptosis is characterized by oxidative cell death, resulting from massive accumulation of lipid reactive oxygen species (ROS), caused by dysregulation of the intracellular iron homeostasis (10). Recent reports have demonstrated a close association between ferroptosis and pathophysiology of multiple diseases (11-13). Hence, modulating ferroptosis to regulate development and progression of serious diseases has become a hot topic in academic research. Indeed, activation of ferroptosis-inducing agents was shown to strongly inhibit proliferation of renal cell carcinoma (14), pancreatic cancer (15), and liver cancer (16). Therefore, the search for ferroptosis-related biomarkers in predicting early diagnosis and prognosis of oncology patients is of utmost importance.

Currently, genome sequencing technology is the chief technique for identification of tumor related biomarkers (17). Long non-coding RNAs (lncRNAs) comprise more than $80 \%$ of the human transcriptome (18). They play a key role in various physiopathological processes by regulating $\mathrm{mRNA}$ expression (19). Multiple evidences suggest a strong link between lncRNAs and tumor development $(20,21)$. Hence, lncRNAs may have potential in the diagnosis and treatment of numerous tumors. Till date, ferroptosis-related lncRNAs have not been studied extensively. Here, we investigated the role of ferroptosis-related lncRNAs in the prognosis of WT patients. We analyzed gene sequencing information from $124 \mathrm{WT}$ and 6 healthy tissue samples and constructed a prognostic signature containing 12 ferroptosis-related lncRNAs. In addition, we evaluated the predictability and immunologic characteristics of the ferroptosisrelated lncRNAs signature. Finally, we proposed potential therapeutic targets for improvements in WT management.

We present the following article in accordance with the TRIPOD reporting checklist (available at https://dx.doi. org/10.21037/tp-21-211).

\section{Methods}

\section{Data collection and processing}

RNA sequencing data from 124 WT and 6 healthy tissue samples and corresponding clinical reports were downloaded from the TARGET database, with the TCGA data portal (https://portal.gdc.camcer.gov/; Data Release 28.0; release time: February 02, 2021). This study abided by the publication criteria provided by TCGA and, therefore, did not require ethics committee approval. To ensure accuracy in analysis, we selected sequencing data from primary tumor tissues for subsequent analysis. Relevant clinical data are summarized in Table 1 . The study was conducted in accordance with the Declaration of Helsinki (as revised in 2013).

\section{Identification of ferroptosis-related lncRNAs}

We obtained 259 ferroptosis-related genes from the FerrDb15 website (http://www.zhounan.org/ferrdb/) (22) and screened for ferroptosis-related genes within the WT verses healthy sequencing information, collected from the TARGET database. Next, Pearson correlation analysis was employed to assess correlations between ferroptosis-related genes and lncRNAs. Ferroptosis-related lncRNAs with correlation coefficient absolute value $>0.6$ and $\mathrm{P}$ value $<0.05$ was considered closely related.

\section{Generation and verification of the ferroptosis-related IncRNA signature}

The differential expression of ferroptosis-related lncRNAs (DElncRNAs) between the WT and healthy samples were screened using the $\mathrm{R}$ software with the following criteria: $\mid \log _{2}$ fold-change (FC) $\mid>1$ and false discovery rate (FDR) $<0.05$. Next, the DElncRNAs with potential prognostic value were recognized using univariate Cox regression analyses. The identified DElncRNAs were then analyzed 
Table 1 Corresponding clinical features of 124 patients with Wilms tumor

\begin{tabular}{|c|c|c|}
\hline \multirow{2}{*}{ Items } & \multicolumn{2}{|c|}{ Patients, $N=124$} \\
\hline & $\mathrm{N}$ & $\%$ \\
\hline \multicolumn{3}{|l|}{ Age } \\
\hline$<5$ & 79 & 63.710 \\
\hline$\geq 5$ & 45 & 36.290 \\
\hline \multicolumn{3}{|l|}{ Gender } \\
\hline Male & 54 & 43.548 \\
\hline Female & 70 & 56.452 \\
\hline \multicolumn{3}{|l|}{ Race } \\
\hline White & 92 & 74.194 \\
\hline Non-White & 32 & 25.806 \\
\hline \multicolumn{3}{|l|}{ Tumor stage } \\
\hline Stage I & 16 & 12.903 \\
\hline Stage II & 49 & 39.516 \\
\hline Stage III & 46 & 37.097 \\
\hline Stage IV & 13 & 10.484 \\
\hline \multicolumn{3}{|c|}{ Histologic classification } \\
\hline FHWT & 42 & 33.871 \\
\hline DAWT & 82 & 66.129 \\
\hline \multicolumn{3}{|c|}{ Survival status } \\
\hline Alive & 74 & 59.677 \\
\hline Dead & 50 & 40.323 \\
\hline
\end{tabular}

with the Lasso-penalized Cox regression analysis to generate the ferroptosis-related lncRNA signature. The formula used was as follows: Risk score $=\left(\operatorname{expression}_{\operatorname{lncRNA1}} \times \beta_{\text {lncRNA1 }}\right)+$ $\left(\operatorname{expression}_{\mathrm{lncRNA} 2} \times \beta_{\mathrm{lncRNA2}}\right)+\ldots+\left(\right.$ expression $\left._{\mathrm{lncRNAn}} \times \beta_{\mathrm{lncRNAn}}\right)$. The patients were then assigned to one of two groups, low-risk or high-risk, according to their median risk score. Kaplan-Meier analysis and ROC curves were then employed to determine predictive power of the signature model.

Next, we analyzed the clinical characteristics (rank sum test) and predictability independence (Cox regression analysis) of the model. Additionally, we generated a nomogram of clinical characteristics and ferroptosis-related lncRNA signature to individualize the estimated 1-, 3-, 5 -year survival of WT patients. Lastly, concordance index (C-index) and calibration curves were employed to assess the prediction accuracy of the nomogram.

\section{Construction of the lncRNA-mRNA co-expression network} and functional enrichment analysis

The mRNAs significantly associated with ferroptosisrelated lncRNAs were recognized with Pearson correlation coefficient (absolute value $>0.3$ at $\mathrm{P}<0.05$ ). Next, Cytoscape was employed to generate the lncRNA-mRNA coexpression network. Gene Ontology (GO) and Kyoto Encyclopedia of Genes and Genomes (KEGG) pathway enrichment mRNA analyses of the IncRNA-mRNA coexpression network were conducted with DAVID (https:// david.ncifcrf.gov/) and KOBAS 3.0 (http://kobas.cbi.pku. edu.cn/kobas3/), respectively. The results of the analyses were visualized using $\mathrm{R}$ software.

\section{Gene set enrichment analysis}

The gene set enrichment analysis (GSEA) was utilized to investigate the differences in biological characteristics between the low- and high-risk groups. The analysis based on 1,000 random permutations, and $\mathrm{P}$ value $<0.05, \mathrm{FDR}<0.25$, INES| $>1$ was considered to be significantly enriched.

\section{Analysis of immunologic characteristics}

Immune cells and responses between two groups were assessed with ESTIMATE (23), MCPcounter (24), CIBERSORT (25), and ssGSEA (26) algorithms. The enrichment levels of 16 immune cells and 13 immunologic functions were further quantified by ssGSEA to evaluate the immunologic characteristics of both groups. In addition, we predicted the potential immune checkpoints.

\section{Statistical analysis}

Overall survival (OS) differences between two groups were analyzed using log-rank test. The ssGSEA scores were compared with the Mann-Whitney test. Data analyses were conducted with packages within $\mathrm{R}$ (version 4.0.4). $\mathrm{R}$ and Cytoscape (version 3.8.2) were used for plotting. Lastly, $\mathrm{P}<0.05$ was the significance threshold.

\section{Results}

\section{Identification of prognostically significant ferroptosis- related lncRNAs}

We screened 239 ferroptosis-related mRNAs, in a total of 130 samples (124 WT and 6 healthy samples), with 

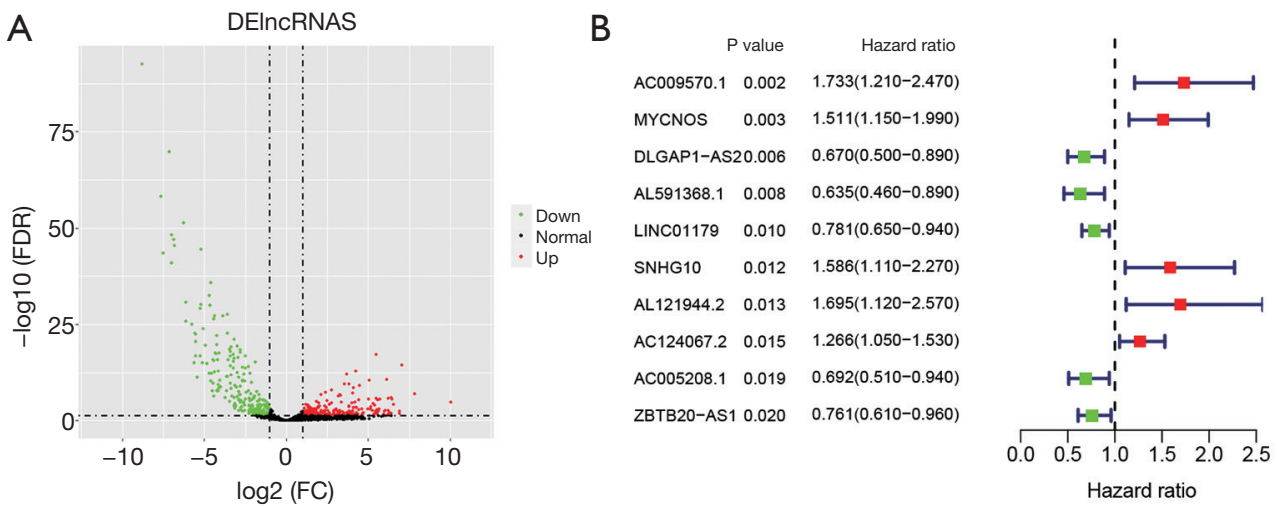

Figure 1 Identification of ferroptosis-related lncRNAs in WT patients. (A) Volcano plot of DElncRNAs. (B) Forest plots of the hazard ratios (HR) of the top 10 ferroptosis-related lncRNAs. WT, Wilms tumor.

information from 259 ferroptosis-related genes (Table S1). We discovered 1,277 ferroptosis-related lncRNAs, using Pearson correlation analysis. Differential expression analysis of these lncRNAs revealed 443 DElncRNAs (Figure 1A, Figure S1). Univariate Cox regression analysis further recognized 26 ferroptosis-related lncRNAs that were closely correlated with OS. The top 10 ferroptosisrelated lncRNAs are illustrated in the forest plot (Figure 1B, Table S2).

\section{Generation of the ferroptosis-related lncRNA signature}

Using Lasso-penalized Cox regression analyses, we analyzed 26 ferroptosis-related lncRNAs with close association with OS (Figure $2 A, 2 B$ ). Based on our analysis, $12 \operatorname{lncRNAs}$ were strong predictors of WT patient prognosis (Table S3). Using this information, we generated a ferroptosis-related lncRNA signature model with the following formula: Risk score $=$ (0.3281× AC007406.1 expression) + (-0.2534× AC005208.1 expression $)+(0.3987 \times$ LINC01770 expression $)+(-0.5637 \times$ DLGAP1-AS2 expression $)+(-0.2012 \times$ AP002761.4 expression $)+(0.3081 \times$ STPG3-AS1 expression $)+(-0.1872 \times$ AC129507.1 expression $)+(0.2002 \times$ AC234772.2 expression $)$ $+(0.3068 \times$ LINC02447 expression $)+(0.4400 \times$ AC009570.1 expression $)+(-0.3948 \times$ ZBTB20-AS1 expression $)+$ (-0.2656x LINC01179 expression).

Next, we separated WT patients into low-risk and high-risk groups, based on the median risk score. Using Kaplan-Meier analysis, we revealed that the high-risk group experienced poor OS, compared to the low-risk group (Figure 2C). The ferroptosis-related lncRNA signaturebased OS predictability was then evaluated by ROC curves.
The AUC values of 1-, 3-, 5-year were $0.775,0.867$, and 0.891 respectively (Figure 2D). Figure $2 E$ illustrates the risk score curve, survival status, and lncRNAs expression data of both groups. As shown, the death cases were concentrated in the high-risk group, relative to the lowrisk group. Moreover, the transcript levels of AC007406.1, LINC01770, STPG3-AS1, AC234772.2, LINC02447, and AC009570.1 were elevated in the high-risk group, relative to the low-risk group. Alternately, the levels of AC005208.1, DLGAP1-AS2, AP002761.4, AC129507.1, ZBTB20-AS1, and LINC01179 were diminished in the high- vs. the lowrisk group.

We also examined the association between the ferroptosisrelated lncRNA signature and clinical characteristics, such as, age, gender, race, stage, and histological classification. Based on our analysis, there was a strong correlation between the ferroptosis-related lncRNA signature and tumor stage and histological classification (Figure 3A). This suggests the possibility of using the ferroptosis-related lncRNA signature for dentification of tumor progression. To test whether this signature can independently predict WT patient prognosis, we further performed univariate and multivariate Cox regression analyses. Univariate Cox regression analysis revealed that the gender, tumor stage, and risk score strongly predicted WT patient prognosis (Figure 3B, Table S4) whereas multivariate Cox regression analysis revealed that tumor stage and risk scores were stand-alone estimators of WT patient prognosis (Figure 3C, Table S5). Furthermore, we plotted ROC curves of clinical characteristics verses ferroptosis-related lncRNA signature and demonstrated that the risk score AUC value (0.775) was far greater than other clinical characteristics (Figure 3D). 

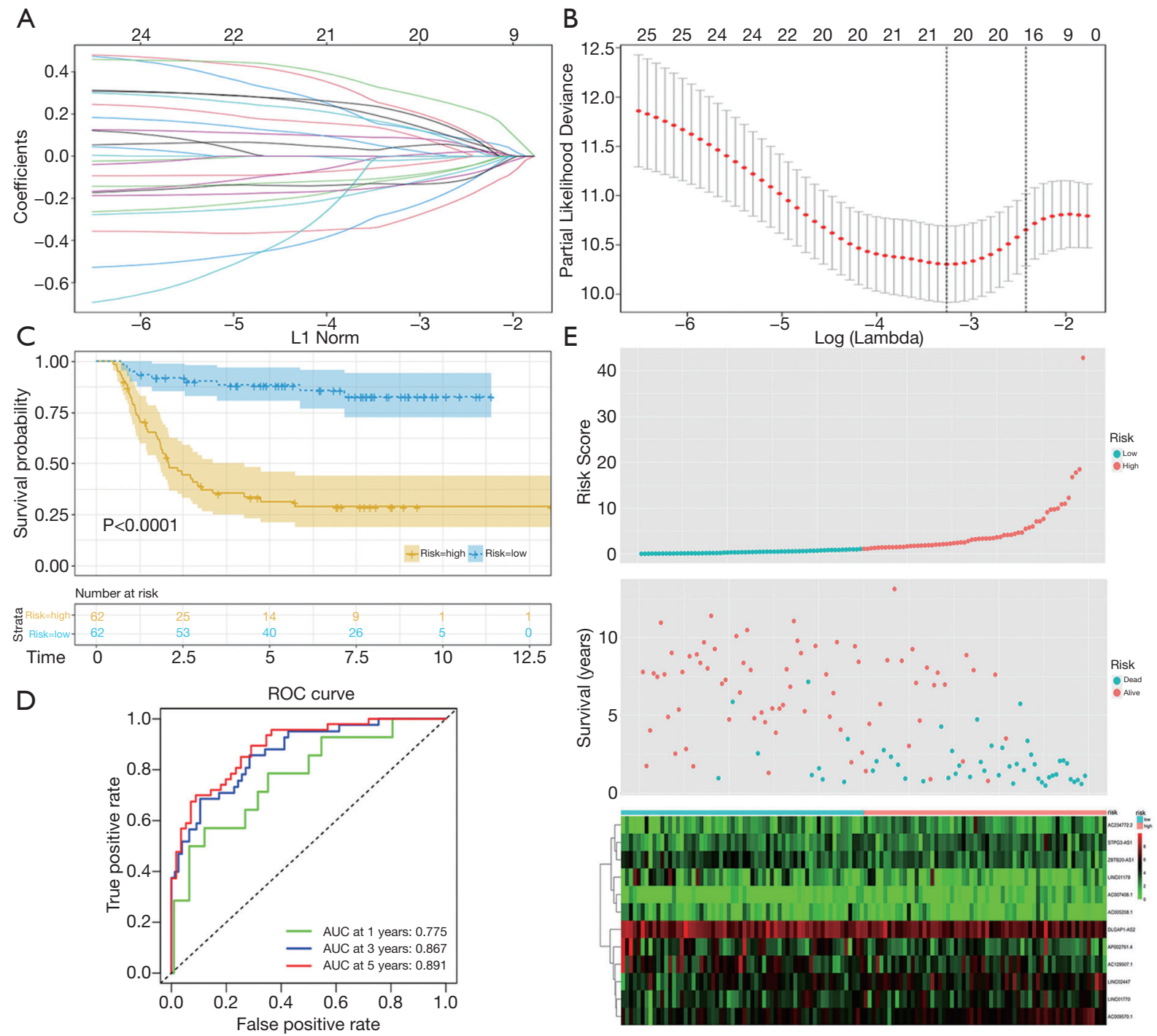

Figure 2 Prognosis analysis of the ferroptosis-related lncRNA signature in TARGET database. (A) LASSO coefficient profiles of the 26 ferroptosis-related lncRNAs correlated with OS; (B) Confidence interval under each lambda; (C) Kaplan-Meier curve and (D) ROC curve of the ferroptosis-related lncRNA signature in WT patients; (E) Prognostic classifier analyses (risk scores, survival status and lncRNAs expression) in distinguishing patients into low- and high-risk groups. WT, Wilms tumor; OS, overall survival.

\section{Establishment of individualized prognostic prediction nomogram}

To increase the accuracy of WT patient prognosis prediction, we generated a nomogram, based on the relationship between the clinical characteristics and ferroptosis-related lncRNA signature (Figure 4A). The nomogram had a $\mathrm{C}$-index of 0.836 , which enabled us to estimate the 1-, 3-, and 5-year survival of WT patients. Using calibration curves, we revealed that the predicted survival was very close to the actual survival (Figure $4 B$ ).
Therefore, we confirmed the accuracy of the ferroptosisrelated individualized prognostic prediction nomogram.

\section{Construction of the lncRNA-mRNA co-expression network}

We also constructed a lncRNA-mRNA co-expression network to illustrate the relationships between RNAs (Figure 5A). This network consisted of 214 lncRNA-mRNA pairs, generated from 12 lncRNAs and 128 mRNAs. The risk characteristics of lncRNAs and mRNAs in the co- 

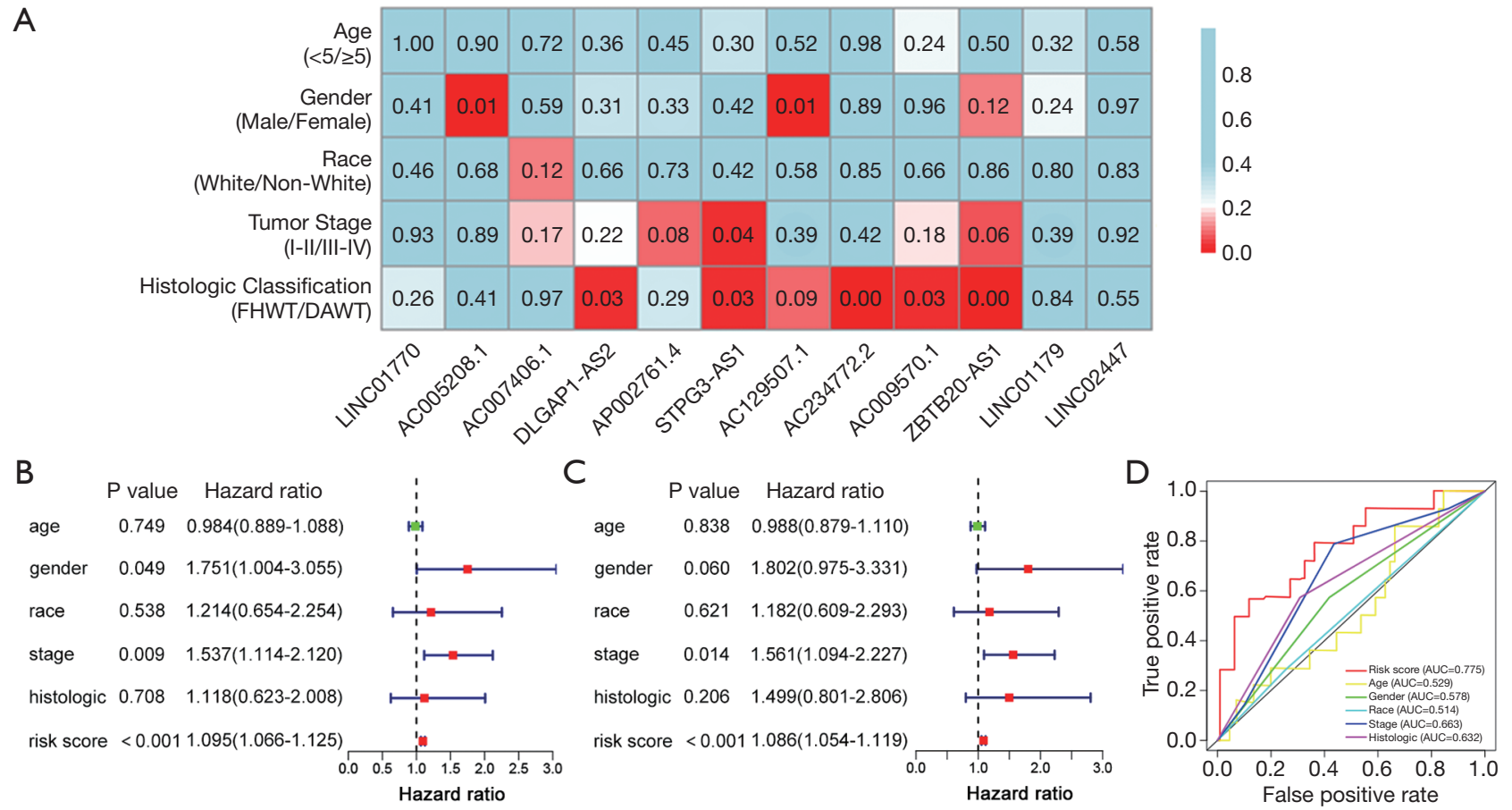

Figure 3 Clinical significance of ferroptosis-related lncRNAs in WT. (A) Correlations between ferroptosis-related lncRNAs and clinical characteristics; (B) Univariate and (C) multivariate Cox regression analysis of clinical characteristics and the signature-based risk score; (D) ROC curve of clinical characteristics and the signature-based risk score. WT, Wilms tumor.

expression network are summarized in the Sankey diagram (Figure 5B). Next, we examined the biological functions of mRNAs within the network, using GO and KEGG enrichment analyses (Figure 5C,5D). Based on our analysis, the biological processes were enriched in the "oxidationreduction process"; the cellular components were enriched in the "cytoplasm"; and the molecular function was mainly enriched in "protein binding". According to the KEGG analysis, the "metabolic pathways" and "pathway in cancer" were most enriched. Interestingly, mRNAs from the lncRNA-mRNA co-expression network were also enriched in immunologic pathways, such as, "Th17 cell differentiation", "T cell receptor signaling pathway", "natural killer cell mediated cytotoxicity", and "B cell receptor signaling pathway". This is indicative of the ferroptosis-related lncRNAs being closely related to tumor immunity.

\section{Gene set enrichment analyses}

GSEA was employed for the analysis of enrichment differences between two groups (Figure 6). We revealed that the low-risk group was enriched in multiple immunerelated biological processes, such as "regulation of substrate adhesion", "positive regulation of endothelial", "regulation of NOTCH signaling pathway", "lymphoid progenitor cell differentiation", and "mature B cell differentiation involved in immune response". It is possible that the low-risk group had better prognosis due to the possible enhancement of immune function. Given these data, the ferroptosis-related lncRNAs may potentially be associated with immune regulation.

\section{Immunologic characteristics of ferroptosis-related lncRNAs}

We further evaluated the relationship between WT patient prognosis and immune status using ESTIMATE, MCPcounter, CIBERSORT, and ssGSEA algorithms (Figure 7). The ssGSEA-based quantitative evaluation of the immunologic characteristics between the two groups revealed that there were marked differences between presence of immune cells and immunologic functions between two groups. In particular, immune cells like B cells, NK cells, Th1 cells, and Treg cells, as well as immunologic 

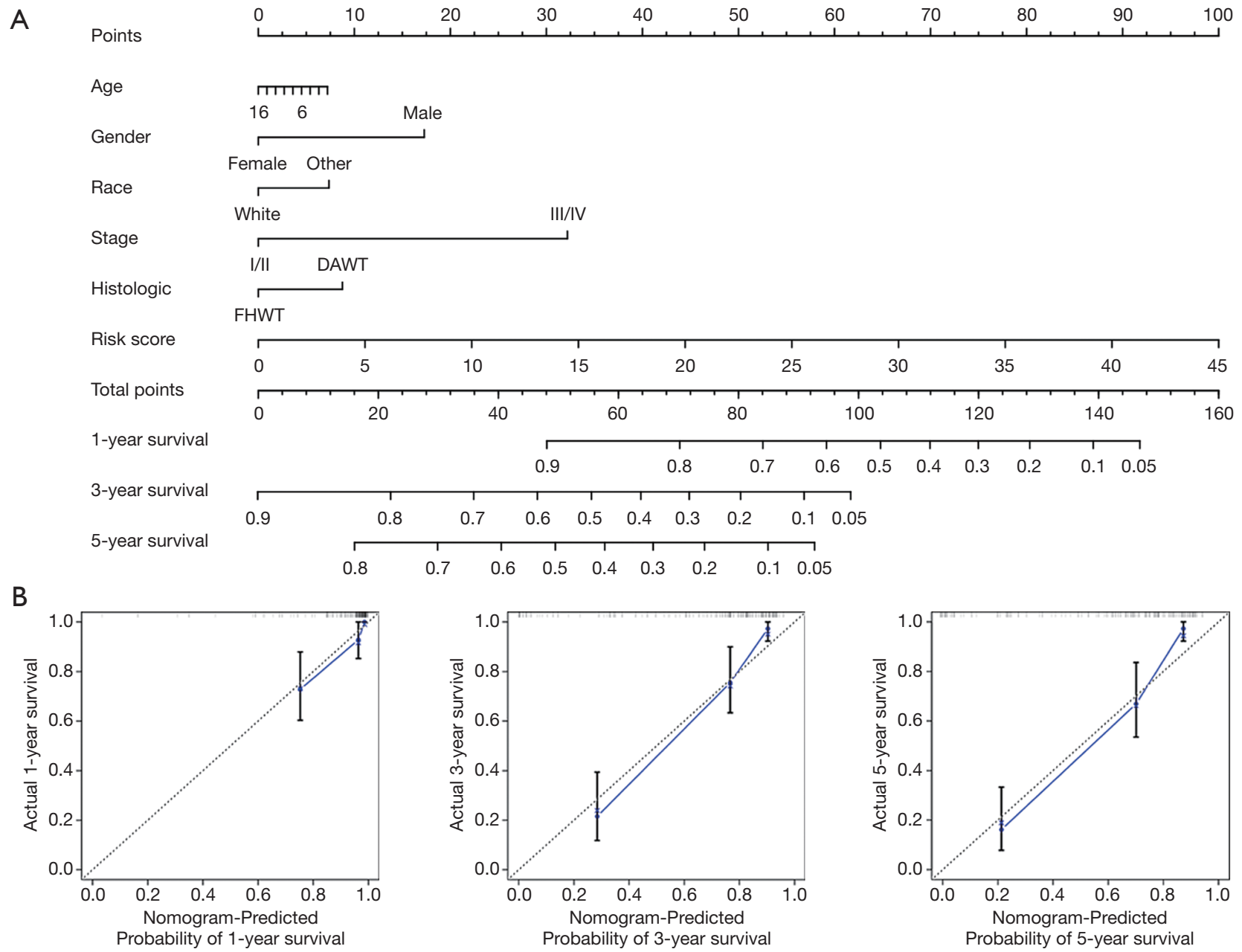

Figure 4 A nomogram of clinical characteristics and ferroptosis-related lncRNAs. (A) Prediction of 1-, 3- and 5-year survival of WT patients based on the nomogram; (B) Calibration curves between actual and predicted survival at 1-, 3- and 5-year.

functions, such as, inflammation-promoting and type I and II IFN responses were vastly different between the two groups (Figure $8 A, 8 B$ ). In addition, we screened immune checkpoints, and distinct differences were observed in the expression of SIRPA, ICOSLG, LAG3, PVRIG, NECTIN1, SIRPB2 between two groups (Figure 8C).

\section{Discussion}

WT is a common urological tumor in children. Despite advances in WT care, tumor stage and postoperative recurrence remain the main factors affecting long-term survival of patients (27). In recent years, ferroptosis has been discovered as a novel form of cell death (8). Moreover, it has been shown to regulate development of numerous tumor cells and suppress tumor sensitivity to chemotherapeutic agents $(28,29)$. Till date, the function of ferroptosis in WT has not been elucidated. Given its intricate relationship in multiple tumors, the identification of ferroptosis-related biomarkers in WT patients is of utmost importance, especially in terms of diagnostic and prognostic evaluation. Furthermore, the search for potential therapeutic targets by revealing ferroptosis-related signaling pathways may help improve anti-tumor therapy for WT patients.

Being ubiquitously expressed in the human transcriptome, lncRNAs are critical to the occurrence and development of tumors (30). Therefore, it is particularly important to explore the ferroptosis-related lncRNAs in WT patients. Here, we systematically evaluated the ferroptosis-related lncRNAs in WT patients and generated a ferroptosis- 
A

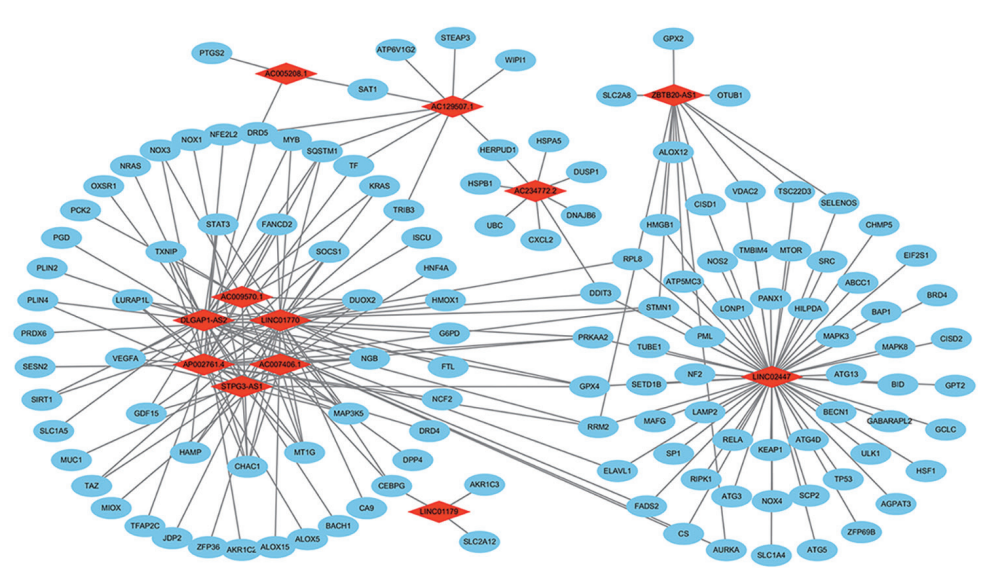

C
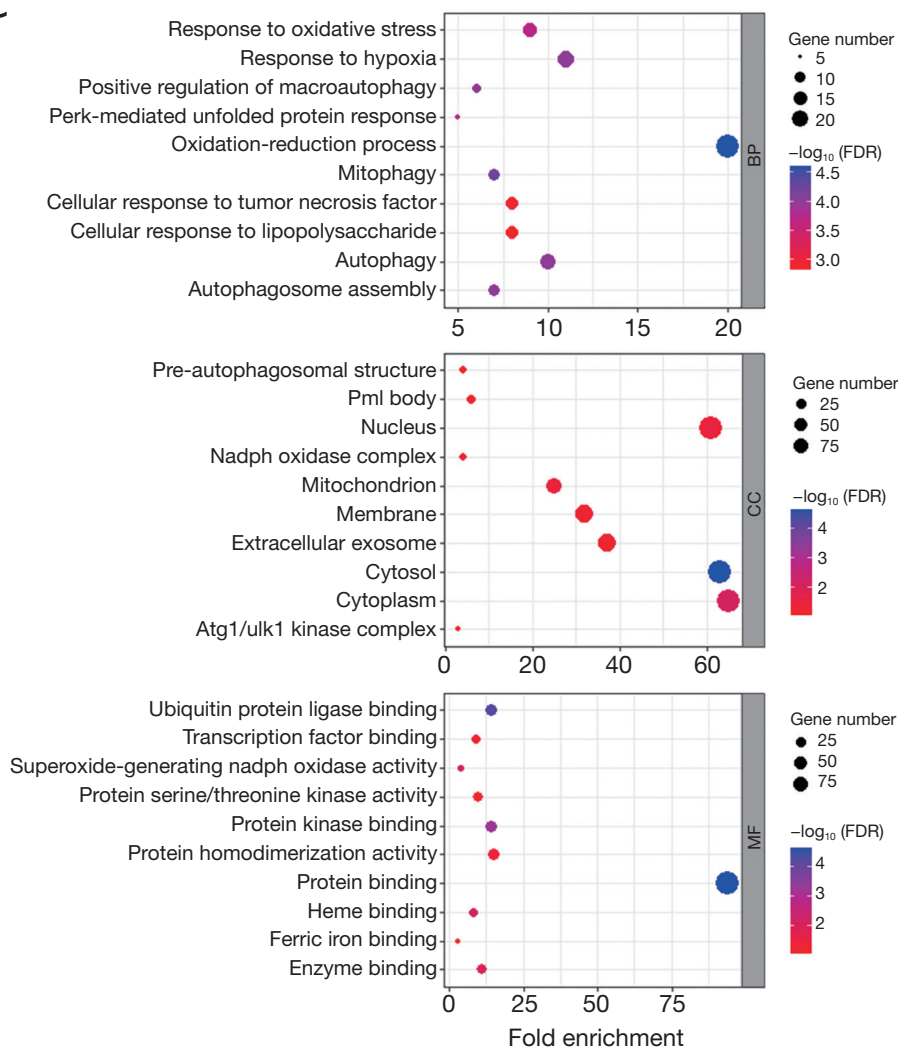

B
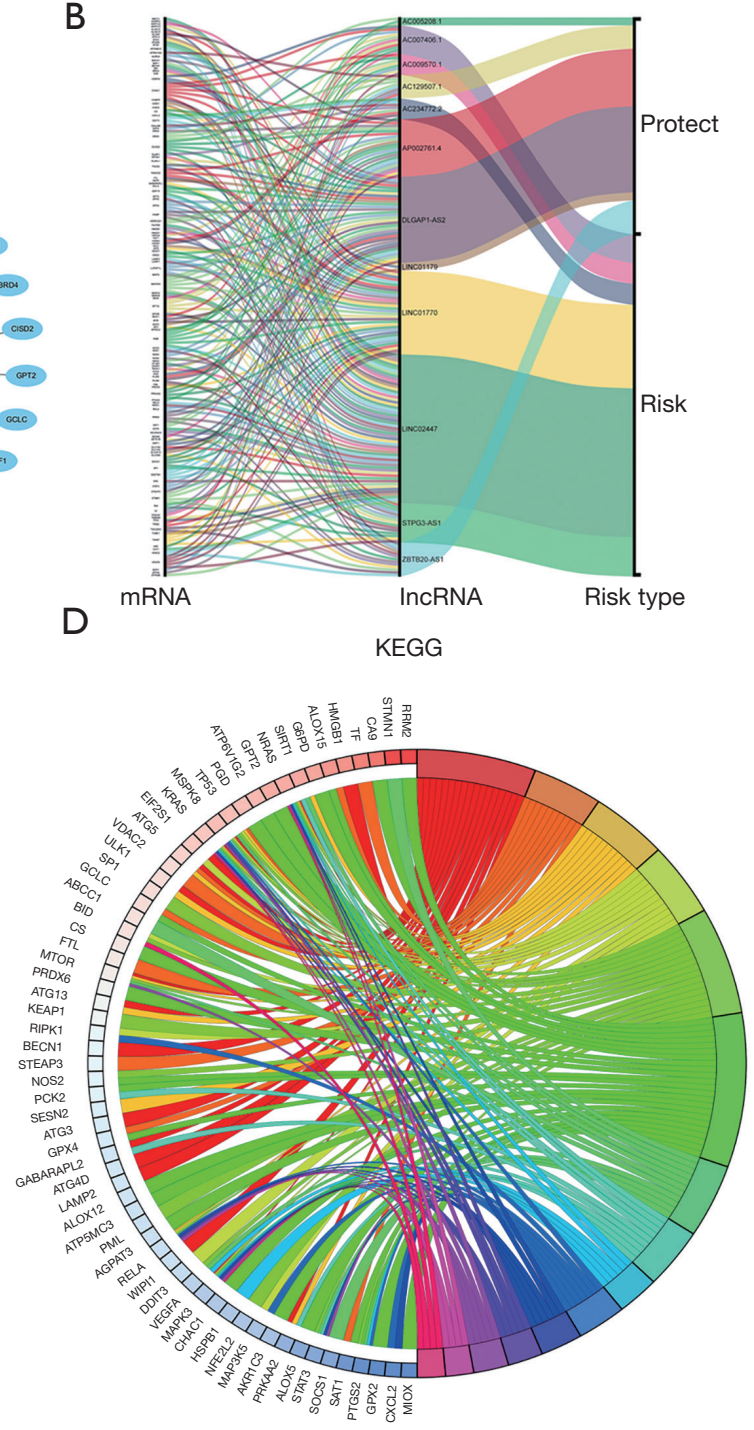

KEGG Pathways

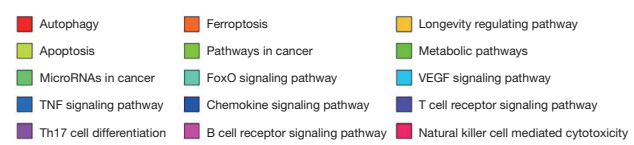

Figure 5 The relationship and functional enrichment between ferroptosis-related lncRNA and mRNA expression. (A) The ferroptosisrelated lncRNA-mRNA co-expression network; (B) The Sankey diagram of RNAs in co-expression network; (C) GO and (D) KEGG analyses of genes included in the co-expression network.

related lncRNA signature for the prediction of patient prognosis. Additionally, we explored correlations between the ferroptosis-related lncRNA signature and immunologic characteristics to identify potential therapeutic targets for the management of WT.

We first identified 12 ferroptosis-related lncRNAs
(AC007406.1, AC005208.1, LINC01770, DLGAP1-AS2, AP002761.4, STPG3-AS1, AC129507.1, AC234772.2, LINC02447, AC009570.1, ZBTB20-AS1 and LINC01179) using univariate Cox and Lasso-penalized Cox regression analyses, and established a ferroptosis-related lncRNA signature model to evaluate prognosis. DLGAP1-AS2 was 


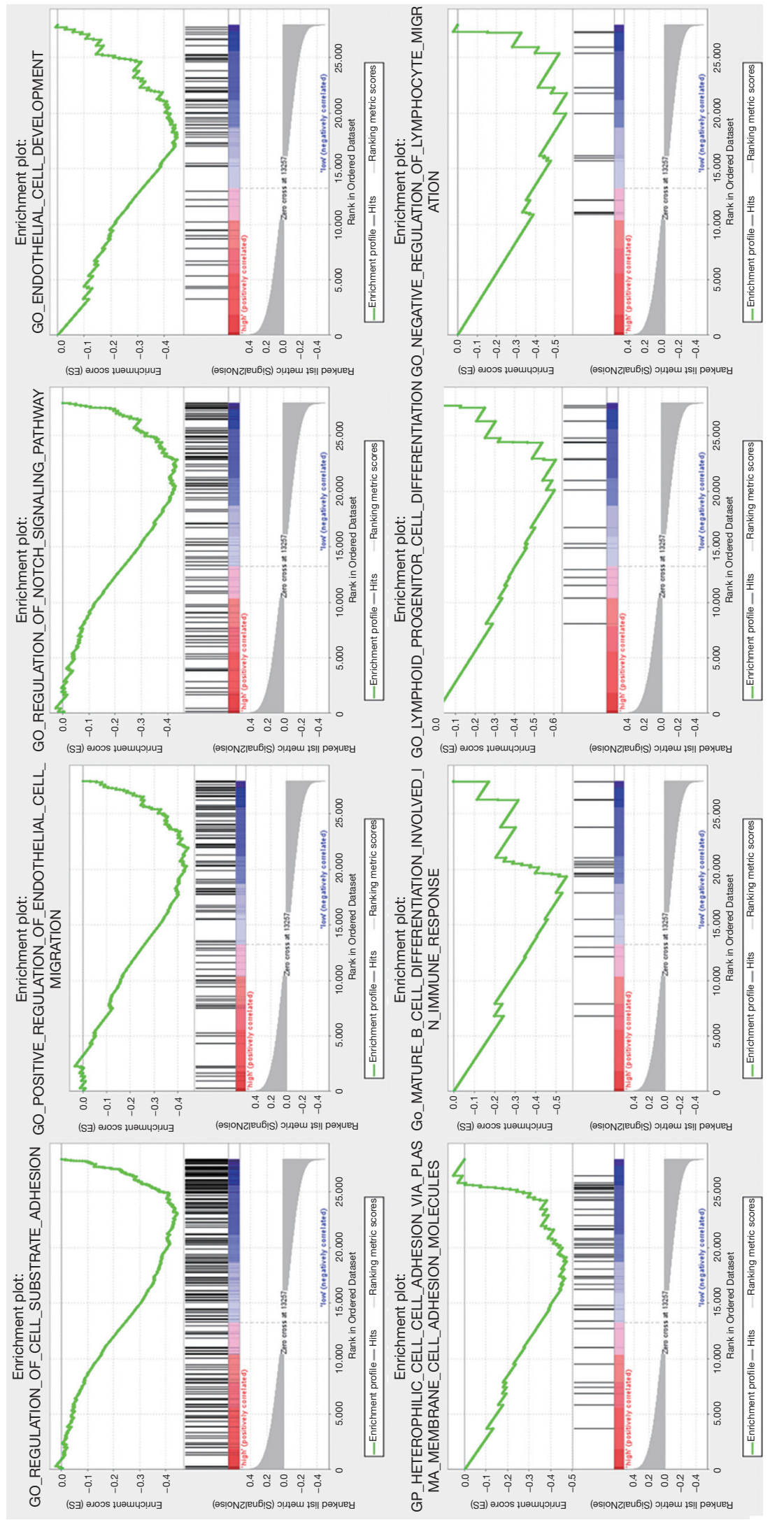

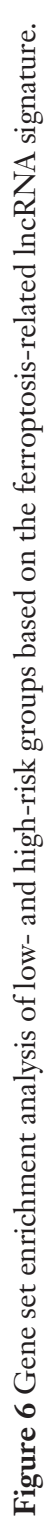




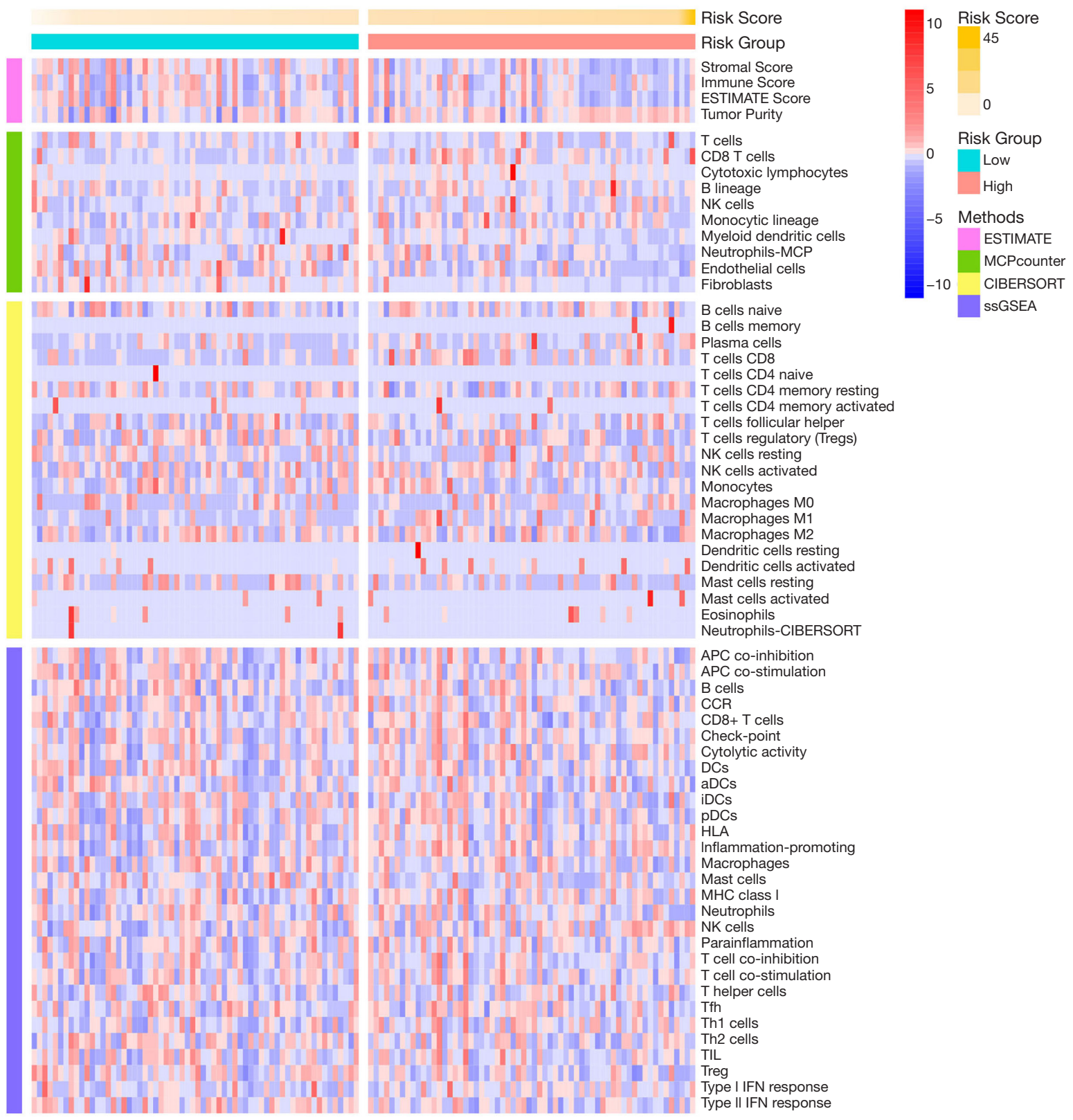

Figure 7 Heatmap of immune status in low- and high-risk groups based on ESTIMATE, MCPcounter, CIBERSORT and ssGSEA algorithms.

previously shown to target YAPP1 and promote glioma tumorigenesis (31). In addition, it was also shown to promote hepatocellular carcinoma and cholangiocarcinoma progression via regulation of miR-154 and miR-505, respectively $(32,33)$. However, other ferroptosis-related lncRNAs have not been studied in relation to tumors, and, therefore, requires additional investigation.

We next tested the accuracy of our signature model's predictive power, using Kaplan-Meier and ROC curves. The AUC values for our model predicting the 1-, 3-, and 5 -year OS rate were $0.775,0.867$ and 0.891 respectively. Given that AUC values $>0.70$ indicate excellent performance, our signature model had high predictability value. Moreover, our predictability value was better than a WT risk evaluation model constructed by Ren et al. using thigh-riskee lncRNAs (34). Next, we explored correlations 
A

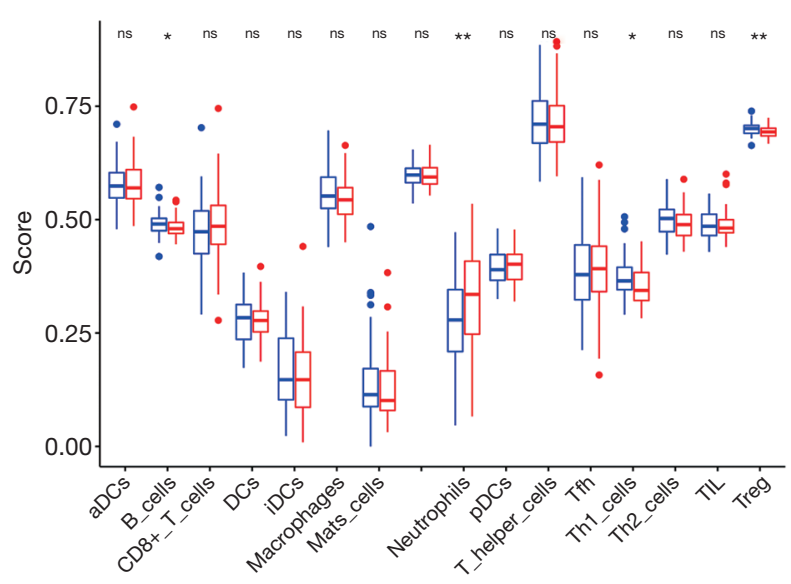

B

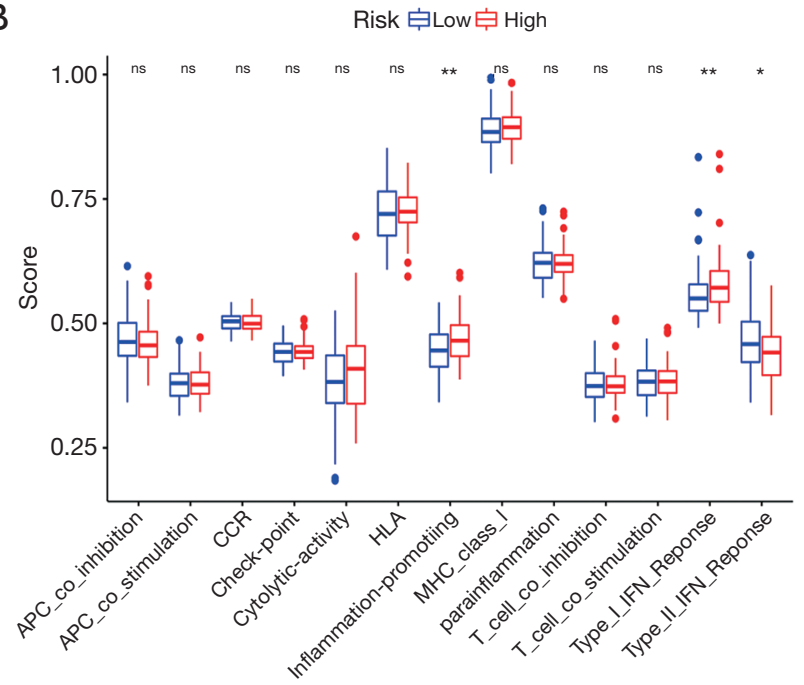

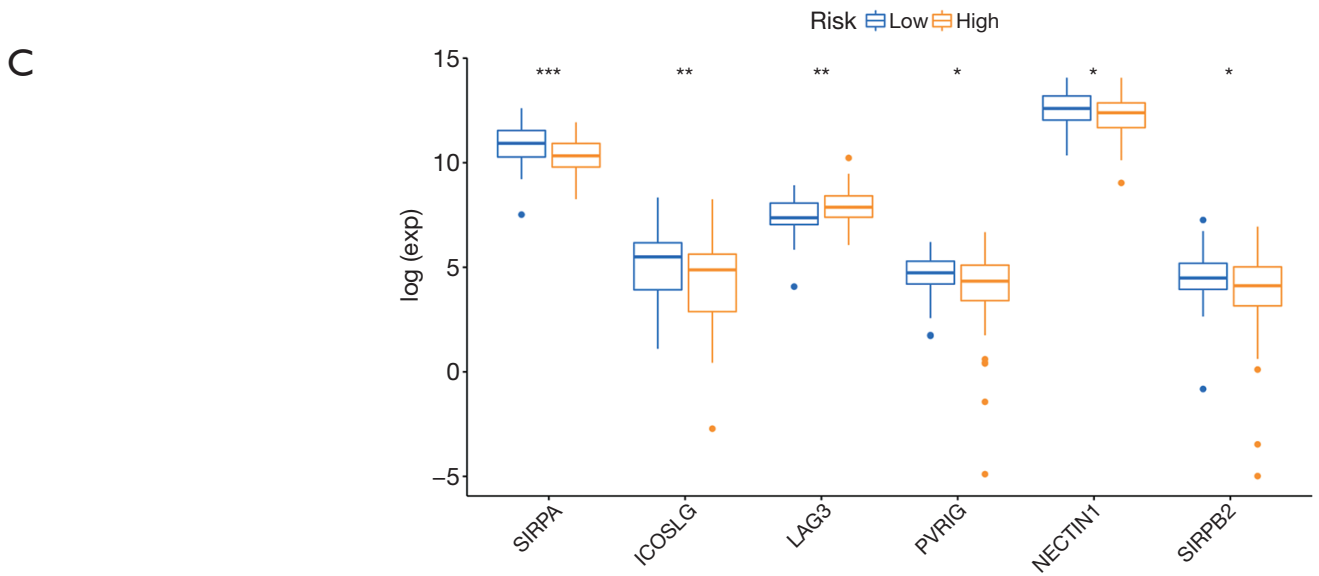

Figure 8 Immunological characteristics of the ferroptosis-related lncRNA signature. Boxplots of (A) immune cells and (B) immune-related functions between low- and high-risk groups by ssGSEA scores; (C) Boxplots of immune checkpoints expression of two groups. *, $\mathrm{P}<0.05$; **, $\mathrm{P}<0.01 ; * * *, \mathrm{P}<0.001$ (adjusted $\mathrm{P}$ values).

between the lncRNAs signature model and clinical characteristics, and found tumor stage and histological classification to be strongly correlated with the lncRNAs signature. This implies that the risk score has great potential for evaluating the prognosis of WT patients. We further tested this correlation using multivariate Cox analysis and showed that the risk score can be an efficient stand-alone estimator of WT patient prognosis.

Nomograms are widely used in oncology medicine as formulative diagrams to understand the relationship between a patient's individualized survival status and clinical variables (35). We, therefore, generated a nomogram based on the risk score and clinical variables like age, gender, race, stage, and histologic classification. Through the nomogram, we confirmed that the tumor stage and risk score are the main determinants of WT patient prognosis. Generally, a c-index $>0.5$ is considered to have predictive value. Pan et al. constructed a prognostic nomogram for WT patients with a c-index of 7.46 (36). We achieved a higher c-index value of 0.836 , suggesting a better predictive value. Furthermore, calibration curves showed that our predicted survival were similar to actual survival. Collectively, this nomogram demonstrated great potential in predicting long-term survival in WT patients and can be adapted into clinical practice in the future.

LncRNAs exert biological functions by regulating mRNA expression (37). Hence, to delineate the role of lncRNA in WT progression, we generated a lncRNA-mRNA co- 
expression network, using the ferroptosis-related lncRNAs, and analyzed its underlying molecular mechanisms with GO and KEGG analysis. GO analysis revealed that the co-expression network mRNAs were mostly enriched in the oxidation-reduction process and protein binding. It is possible that this is related to the induction of lipid peroxidation after ferroptosis activation (38). On the other hand, the chief enriched KEGG pathways involved metabolic, cancer, and immunologic pathways. Given its enrichment of the immunologic pathway, we hypothesized that ferroptosis-related IncRNAs may regulate tumor immunity. As we expected, GSEA results demonstrated that the low-risk WT samples were enriched in multiple immunologic pathways, further suggesting a link between ferroptosis-related lncRNAs and tumor immunity.

Given the relevance of ferroptosis-related lncRNAs in tumor immunity, we next assessed differences among immune cells and within immunologic functions between two groups using ssGSEA. We demonstrated a marked decrease in B cells, Th1 cells, and Treg cells in the highrisk group, whereas NK cells were remarkably elevated. This is suggestive of the NK cells becoming predominant within the tumor microenvironment of WT high-risk patients. In a study by Sottile et al., reduction in iron and ferritin modulate content in tumor cells was shown to activate NK cells (39), thereby indicating a link between ferroptosis and NK cell activation. Moreover, differential analysis of immunologic functions revealed an increased type I IFN response in the high-risk group, along with a simultaneous decrease in type II response. This may be related to a potential dual mechanism of the type I IFN (40). In addition, the decreased type II IFN response is indicative of poor antigen-presentation in high-risk patients, which may contribute to poor prognosis (41). Recent evidences reveal that abnormal levels of immune checkpoints may be an important factor in cancer development (42). Therefore, we screened immune checkpoints genes for differential expression between the low- and high-risk groups. Based on our analysis, the SIRPA, ICOSLG, LAG3, PVRIG, NECTIN1, and SIRPB2 genes were markedly different between two groups and, therefore, can be used as potential therapeutic targets for the management of WT.

Admittedly, there were several limitations in our study. First, due to the rarity of the disease, we only performed a search of the TARGET database, which was not multicenter validated. Secondly, the TARGET database selected for this study contained only 124 Wilms tumors and 6 healthy tissue samples. In the future, we will collect more data to strengthen our study. Finally, this study was retrospective in nature and based on database analysis. Hence, further clinical confirmation is needed to validate the results in the future.

\section{Conclusions}

In summary, we established a ferroptosis-related lncRNA signature for the prediction of $\mathrm{W}^{\mathrm{T}} \mathrm{T}$ patient prognosis and provided potential therapeutic targets for the treatment of WT. To aid in individualized prognosis, we also generated a nomogram using clinical characteristics. Lastly, we detected a close relationship between ferroptosis-related lncRNAs and tumor immunity, which is impaired in WT patients, and screen for potential immune checkpoints.

\section{Acknowledgments}

Funding: This work was supported by the National Natural Science Foundation of China [81871837, 81572117] and the Specialized Research Fund for Doctoral Programs in Colleges and Universities of China [20132307110007].

\section{Footnote}

Reporting Checklist: The authors have completed the TRIPOD reporting checklist. Available at https://dx.doi. org/10.21037/tp-21-211

Conflicts of Interest: All authors have completed the ICMJE uniform disclosure form (available at https://dx.doi. org/10.21037/tp-21-211). ZL reports that the manuscript was supported by the National Natural Science Foundation of China (81871837 and 81572117) and the Specialized Research Fund for Doctoral Programs in Colleges and Universities of China (20132307110007). The other authors have no conflicts of interest to declare.

Ethical Statement: The authors are accountable for all aspects of the work in ensuring that questions related to the accuracy or integrity of any part of the work are appropriately investigated and resolved. The study was conducted in accordance with the Declaration of Helsinki (as revised in 2013).

Open Access Statement: This is an Open Access article distributed in accordance with the Creative Commons Attribution-NonCommercial-NoDerivs 4.0 International 
License (CC BY-NC-ND 4.0), which permits the noncommercial replication and distribution of the article with the strict proviso that no changes or edits are made and the original work is properly cited (including links to both the formal publication through the relevant DOI and the license). See: https://creativecommons.org/licenses/by-nc-nd/4.0/.

\section{References}

1. Hohenstein P, Pritchard-Jones K, Charlton J. The yin and yang of kidney development and Wilms' tumors. Genes Dev 2015;29:467-82.

2. Charlton J, Irtan S, Bergeron C, et al. Bilateral Wilms tumour: a review of clinical and molecular features. Expert Rev Mol Med 2017;19:e8.

3. Davidoff AM. Wilms tumor. Adv Pediatr 2012;59:247-67.

4. Szychot E, Apps J, Pritchard-Jones K. Wilms' tumor: biology, diagnosis and treatment. Transl Pediatr 2014;3:12-24.

5. Breslow NE, Lange JM, Friedman DL, et al. Secondary malignant neoplasms after Wilms tumor: an international collaborative study. Int J Cancer 2010;127:657-66.

6. Geller JI. Current standards of care and future directions for "high-risk" pediatric renal tumors: Anaplastic Wilms tumor and Rhabdoid tumor. Urol Oncol 2016;34:50-6.

7. Mavinkurve-Groothuis AM, van den Heuvel-Eibrink MM, Tytgat GA, et al. Treatment of relapsed Wilms tumour (WT) patients: experience with topotecan. A report from the SIOP Renal Tumour Study Group (RTSG). Pediatr Blood Cancer 2015;62:598-602.

8. Li J, Cao F, Yin HL, et al. Ferroptosis: past, present and future. Cell Death Dis 2020;11:88.

9. Dixon SJ, Lemberg KM, Lamprecht MR, et al. Ferroptosis: an iron-dependent form of nonapoptotic cell death. Cell 2012;149:1060-72.

10. Hirschhorn T, Stockwell BR. The development of the concept of ferroptosis. Free Radic Biol Med 2019;133:130-43.

11. Hambright WS, Fonseca RS, Chen L, et al. Ablation of ferroptosis regulator glutathione peroxidase 4 in forebrain neurons promotes cognitive impairment and neurodegeneration. Redox Biol 2017;12:8-17.

12. Karuppagounder SS, Alin L, Chen Y, et al. $\mathrm{N}$-acetylcysteine targets 5 lipoxygenase-derived, toxic lipids and can synergize with prostaglandin $\mathrm{E} 2$ to inhibit ferroptosis and improve outcomes following hemorrhagic stroke in mice. Ann Neurol 2018;84:854-72.

13. Martin-Sanchez D, Ruiz-Andres O, Poveda J, et al.
Ferroptosis, but Not Necroptosis, Is Important in Nephrotoxic Folic Acid-Induced AKI. J Am Soc Nephrol 2017;28:218-29.

14. Miess H, Dankworth B, Gouw AM, et al. The glutathione redox system is essential to prevent ferroptosis caused by impaired lipid metabolism in clear cell renal cell carcinoma. Oncogene 2018;37:5435-50.

15. Eling N, Reuter L, Hazin J, et al. Identification of artesunate as a specific activator of ferroptosis in pancreatic cancer cells. Oncoscience 2015;2:517-32.

16. Louandre C, Marcq I, Bouhlal H, et al. The retinoblastoma $(\mathrm{Rb})$ protein regulates ferroptosis induced by sorafenib in human hepatocellular carcinoma cells. Cancer Lett 2015;356:971-7.

17. Pareek CS, Smoczynski R, Tretyn A. Sequencing technologies and genome sequencing. J Appl Genet 2011;52:413-35.

18. Alexander RP, Fang G, Rozowsky J, et al. Annotating non-coding regions of the genome. Nat Rev Genet 2010;11:559-71.

19. Qian X, Zhao J, Yeung PY, et al. Revealing lncRNA Structures and Interactions by Sequencing-Based Approaches. Trends Biochem Sci 2019;44:33-52.

20. Peng WX, Koirala P, Mo YY. LncRNA-mediated regulation of cell signaling in cancer. Oncogene 2017;36:5661-7.

21. Bhan A, Soleimani M, Mandal SS. Long Noncoding RNA and Cancer: A New Paradigm. Cancer Res 2017;77:3965-81.

22. Zhou N, Bao J. FerrDb: a manually curated resource for regulators and markers of ferroptosis and ferroptosis-disease associations. Database (Oxford) 2020;2020:baaa021.

23. Yoshihara K, Shahmoradgoli M, Martínez E, et al. Inferring tumour purity and stromal and immune cell admixture from expression data. Nat Commun 2013;4:2612.

24. Dienstmann R, Villacampa G, Sveen A, et al. Relative contribution of clinicopathological variables, genomic markers, transcriptomic subtyping and microenvironment features for outcome prediction in stage II/III colorectal cancer. Ann Oncol 2019;30:1622-9.

25. Chen B, Khodadoust MS, Liu CL, et al. Profiling Tumor Infiltrating Immune Cells with CIBERSORT. Methods Mol Biol 2018;1711:243-59.

26. Yi M, Nissley DV, McCormick F, et al. ssGSEA scorebased Ras dependency indexes derived from gene expression data reveal potential Ras addiction mechanisms 
with possible clinical implications. Sci Rep 2020;10:10258.

27. Routh JC, Grundy PE, Anderson JR, et al. B7-h1 as a biomarker for therapy failure in patients with favorable histology Wilms tumor. J Urol 2013;189:1487-92.

28. Mou Y, Wang J, Wu J, et al. Ferroptosis, a new form of cell death: opportunities and challenges in cancer. J Hematol Oncol 2019;12:34.

29. Xu T, Ding W, Ji X, et al. Molecular mechanisms of ferroptosis and its role in cancer therapy. J Cell Mol Med 2019;23:4900-12.

30. Li J, Meng H, Bai Y, et al. Regulation of lncRNA and Its Role in Cancer Metastasis. Oncol Res 2016;23:205-17.

31. Miao W, Li N, Gu B, et al. LncRNA DLGAP1-AS2 modulates glioma development by up-regulating YAP1 expression. J Biochem 2020;167:411-8.

32. Chen K, Zhang Z, Yu A, et al. lncRNA DLGAP1-AS2 Knockdown Inhibits Hepatocellular Carcinoma Cell Migration and Invasion by Regulating miR-154-5p Methylation. Biomed Res Int 2020;2020:6575724.

33. Liu Z, Pan L, Yan X, et al. The long noncoding RNA DLGAP1-AS2 facilitates cholangiocarcinoma progression via miR-505 and GALNT10. FEBS Open Bio 2021;11:413-22.

34. Ren P, Hu M. A three long non-coding RNA signature to improve survival prediction in patients with Wilms' tumor. Oncol Lett 2019;18:6164-70.

Cite this article as: Liu H, Zhang M, Zhang T, Shi M, Lu W, Yang S, Cui Q, Li Z. Identification of a ferroptosis-related lncRNA signature with prognosis for Wilms tumor. Transl Pediatr 2021;10(10):2418-2431. doi: 10.21037/tp-21-211
35. Balachandran VP, Gonen M, Smith JJ, et al. Nomograms in oncology: more than meets the eye. Lancet Oncol 2015;16:e173-80.

36. Pan Z, You H, Bu Q, et al. Development and validation of a nomogram for predicting cancer-specific survival in patients with Wilms' tumor. J Cancer 2019;10:5299-305.

37. Zhang J, Le TD, Liu L, et al. Inferring and analyzing module-specific lncRNA-mRNA causal regulatory networks in human cancer. Brief Bioinform 2019;20:1403-19.

38. Stockwell BR, Friedmann Angeli JP, Bayir H, et al. Ferroptosis: A Regulated Cell Death Nexus Linking Metabolism, Redox Biology, and Disease. Cell 2017;171:273-85.

39. Sottile R, Federico G, Garofalo C, et al. Iron and Ferritin Modulate MHC Class I Expression and NK Cell Recognition. Front Immunol 2019;10:224.

40. Lee AJ, Ashkar AA. The Dual Nature of Type I and Type II Interferons. Front Immunol 2018;9:2061.

41. Alspach E, Lussier DM, Schreiber RD. Interferon $\gamma$ and Its Important Roles in Promoting and Inhibiting Spontaneous and Therapeutic Cancer Immunity. Cold Spring Harb Perspect Biol 2019;11:a028480.

42. Zhang Y, Zheng J. Functions of Immune Checkpoint Molecules Beyond Immune Evasion. Adv Exp Med Biol 2020;1248:201-26. 


\section{Supplementary}

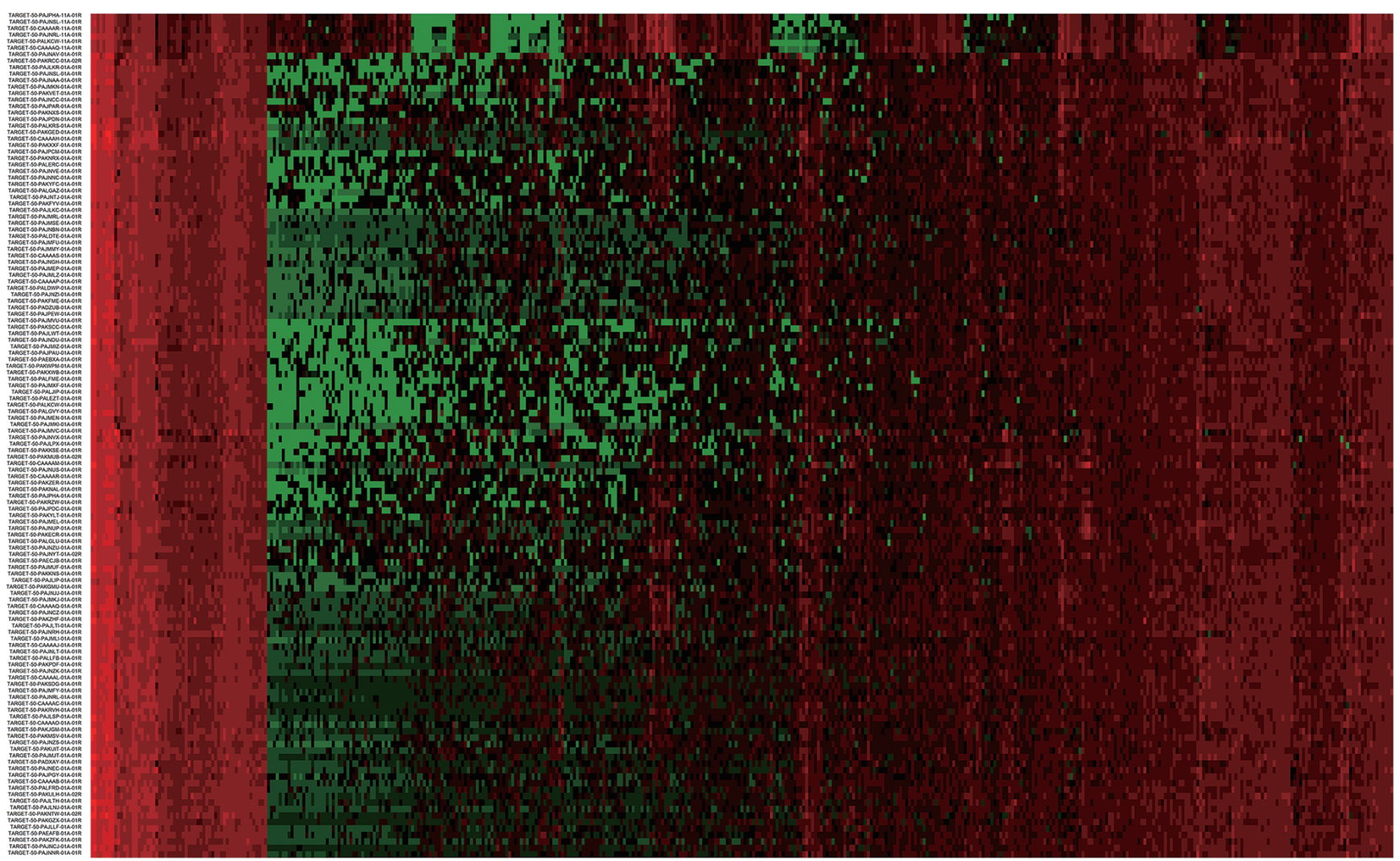

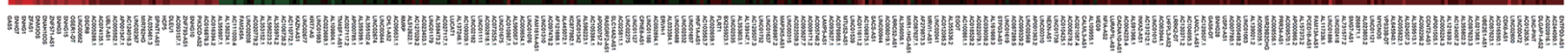

Figure S1 Heatmap of DElncRNAs. 
Table S1 239 ferroptosis-related mRNAs in WT patients

\begin{tabular}{|c|c|c|c|c|c|c|c|}
\hline RPL8 & GLS2 & EGFR & TLR4 & MT1G & CA9 & SLC1A4 & IL33 \\
\hline ATP5MC3 & CARS1 & MAPK1 & ATM & CISD1 & PLIN2 & TXNIP & STEAP3 \\
\hline CS & ALOX5 & BID & YY1AP1 & FANCD2 & AlFM2 & VLDLR & DRD5 \\
\hline EMC2 & KEAP1 & ZEB1 & EGLN2 & HSPA5 & LAMP2 & GPT2 & DRD4 \\
\hline NOX1 & ATG5 & CDKN2A & $\mathrm{TAZ}$ & HELLS & PROM2 & LURAP1L & SLC2A1 \\
\hline CYBB & ATG7 & PEBP1 & MTDH & SCD & CHMP5 & SLC7A5 & SLC2A3 \\
\hline NOX3 & NCOA4 & SOCS1 & IDH1 & FADS2 & CHMP6 & HERPUD1 & SLC2A6 \\
\hline NOX4 & ALOX12 & CDO1 & SIRT1 & SRC & CAV1 & ZNF419 & SLC2A8 \\
\hline DUOX2 & ALOX15B & MAPK9 & DNAJB6 & MTOR & DUSP1 & ZFP69B & EIF2AK4 \\
\hline G6PD & ALOXE3 & CHAC1 & $\mathrm{BACH} 1$ & NFS1 & NOS2 & ATP6V1G2 & TFAP2C \\
\hline PGD & PHKG2 & MAPK14 & LONP1 & TP63 & NCF2 & VEGFA & SP1 \\
\hline VDAC2 & ACO1 & PRKAA2 & SLC7A11 & CDKN1A & MT3 & GDF15 & HBA1 \\
\hline PIK3CA & ULK1 & PRKAA1 & GPX4 & ENPP2 & UBC & TUBE1 & NNMT \\
\hline FLT3 & ATG3 & ELAVL1 & AKR1C1 & $\mathrm{FH}$ & ALB & ARRDC3 & PLIN4 \\
\hline SCP2 & ATG4D & BAP1 & AKR1C2 & CISD2 & TXNRD1 & CEBPG & $\mathrm{HIC1}$ \\
\hline TP53 & BECN1 & $\mathrm{ABCC} 1$ & AKR1C3 & CBS & SRXN1 & RGS4 & STMN1 \\
\hline TF & WIPI2 & IFNG & SQSTM1 & PRDX6 & DDIT4 & HSD17B11 & GABPB1 \\
\hline TFRC & SNX4 & ANO6 & NQO1 & SESN2 & ASNS & AGPAT3 & AURKA \\
\hline TFR2 & ATG13 & LPIN1 & FTH1 & NF2 & TSC22D3 & SETD1B & RIPK1 \\
\hline SLC38A1 & ULK2 & HMGB1 & MUC1 & ARNTL & DDIT3 & FTL & PRDX1 \\
\hline SLC1A5 & SAT1 & TNFAIP3 & SLC3A2 & JUN & JDP2 & MAFG & \\
\hline
\end{tabular}


Table S2 The ferroptosis-related lncRNAs

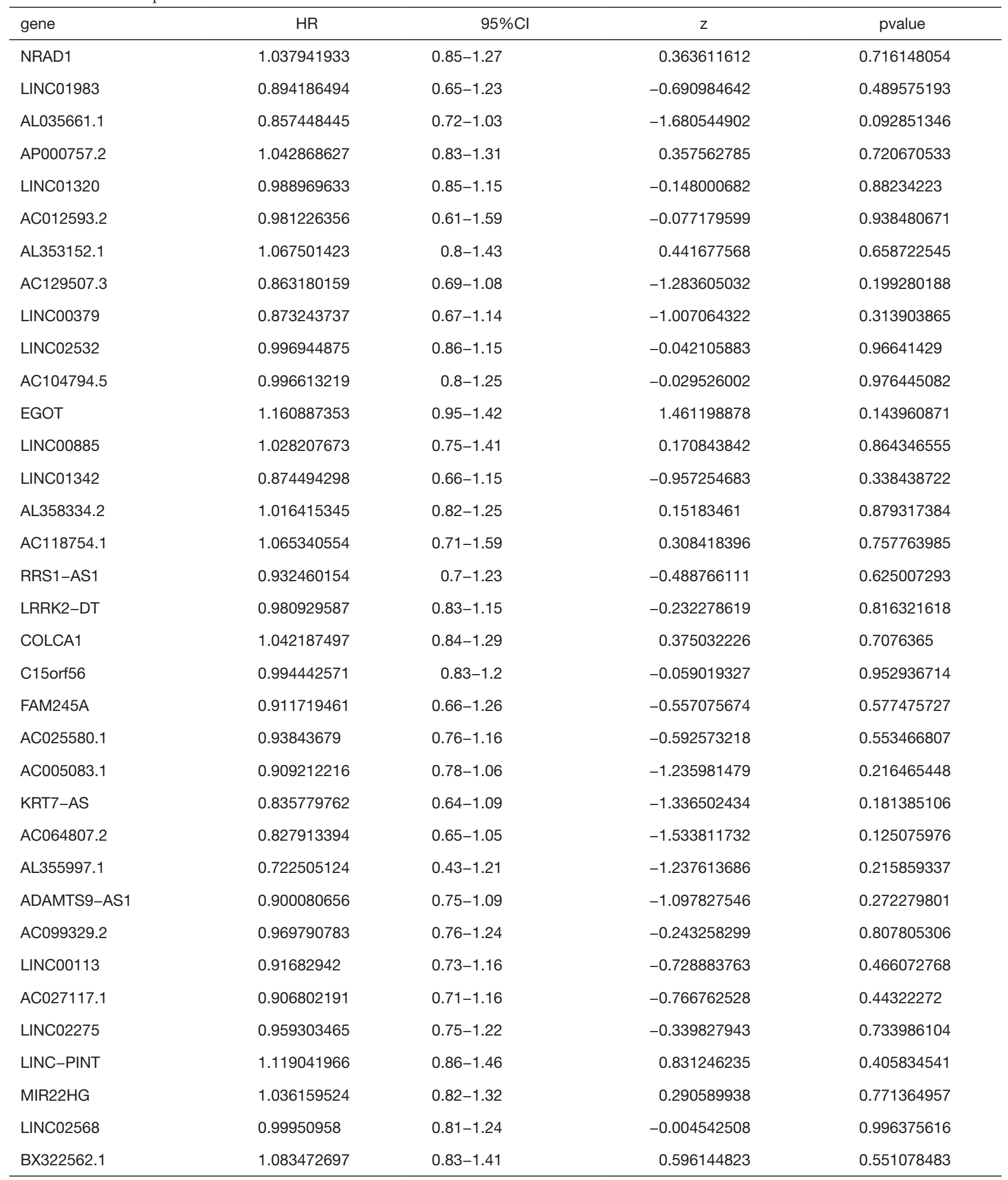

Table S2 (continued) 
Table S2 (continued)

\begin{tabular}{|c|c|c|c|c|}
\hline gene & $\mathrm{HR}$ & $95 \% \mathrm{Cl}$ & z & pvalue \\
\hline LINC01137 & 1.051375631 & $0.81-1.36$ & 0.376419779 & 0.706604844 \\
\hline AC020659.1 & 1.078991648 & $0.87-1.34$ & 0.684147791 & 0.493881846 \\
\hline NR4A1AS & 1.044856613 & $0.84-1.3$ & 0.400154446 & 0.689042765 \\
\hline PRR34-AS1 & 0.881933446 & $0.72-1.08$ & -1.189669231 & 0.234176424 \\
\hline AL023803.2 & 1.032589826 & $0.72-1.48$ & 0.17421492 & 0.861696561 \\
\hline AC020916.1 & 0.913781425 & $0.64-1.3$ & -0.502137418 & 0.615570861 \\
\hline AC092894.1 & 0.932257667 & $0.77-1.13$ & -0.699261561 & 0.484388585 \\
\hline AP000866.2 & 1.041412679 & $0.79-1.37$ & 0.291047001 & 0.771015373 \\
\hline AC130371.2 & 1.03930014 & $0.84-1.28$ & 0.364437757 & 0.715531145 \\
\hline AL162424.1 & 1.098637706 & $0.85-1.42$ & 0.709783456 & 0.47783843 \\
\hline THRB-AS1 & 1.047497608 & $0.84-1.3$ & 0.416205177 & 0.677259872 \\
\hline AC005920.3 & 0.838384039 & $0.53-1.33$ & -0.751106236 & 0.452588723 \\
\hline AC105384.1 & 1.237195967 & $0.96-1.6$ & 1.635993816 & 0.101840882 \\
\hline AL109976.1 & 0.998026061 & $0.81-1.23$ & -0.018186732 & 0.985489887 \\
\hline WDFY3-AS2 & 0.938515142 & $0.59-1.49$ & -0.269643479 & 0.787434548 \\
\hline AC093278.2 & 0.930103118 & $0.68-1.27$ & -0.450331946 & 0.652471108 \\
\hline AL353751.1 & 0.837543594 & $0.64-1.09$ & -1.301510883 & 0.193083642 \\
\hline AC087289.5 & 1.006528828 & $0.75-1.34$ & 0.04433309 & 0.964638896 \\
\hline AL121672.1 & 0.875923578 & $0.69-1.11$ & -1.088486105 & 0.276380567 \\
\hline AP001347.1 & 1.074579863 & $0.79-1.47$ & 0.454757639 & 0.649283609 \\
\hline AC007406.1 & 1.387061186 & $1.01-1.9$ & 2.039801956 & 0.041370055 \\
\hline AC103563.7 & 0.829828832 & $0.71-0.97$ & -2.297957999 & 0.02156418 \\
\hline LINC00472 & 0.938818844 & $0.77-1.15$ & -0.616857193 & 0.537328924 \\
\hline AL390755.1 & 0.777678898 & $0.57-1.07$ & -1.546178481 & 0.122061469 \\
\hline AL031666.1 & 1.304362018 & $0.94-1.81$ & 1.589032244 & 0.112053113 \\
\hline C5orf64-AS1 & 1.052660952 & $0.72-1.54$ & 0.262602546 & 0.792856937 \\
\hline
\end{tabular}

Table S2 (continued) 
Table S2 (continued)

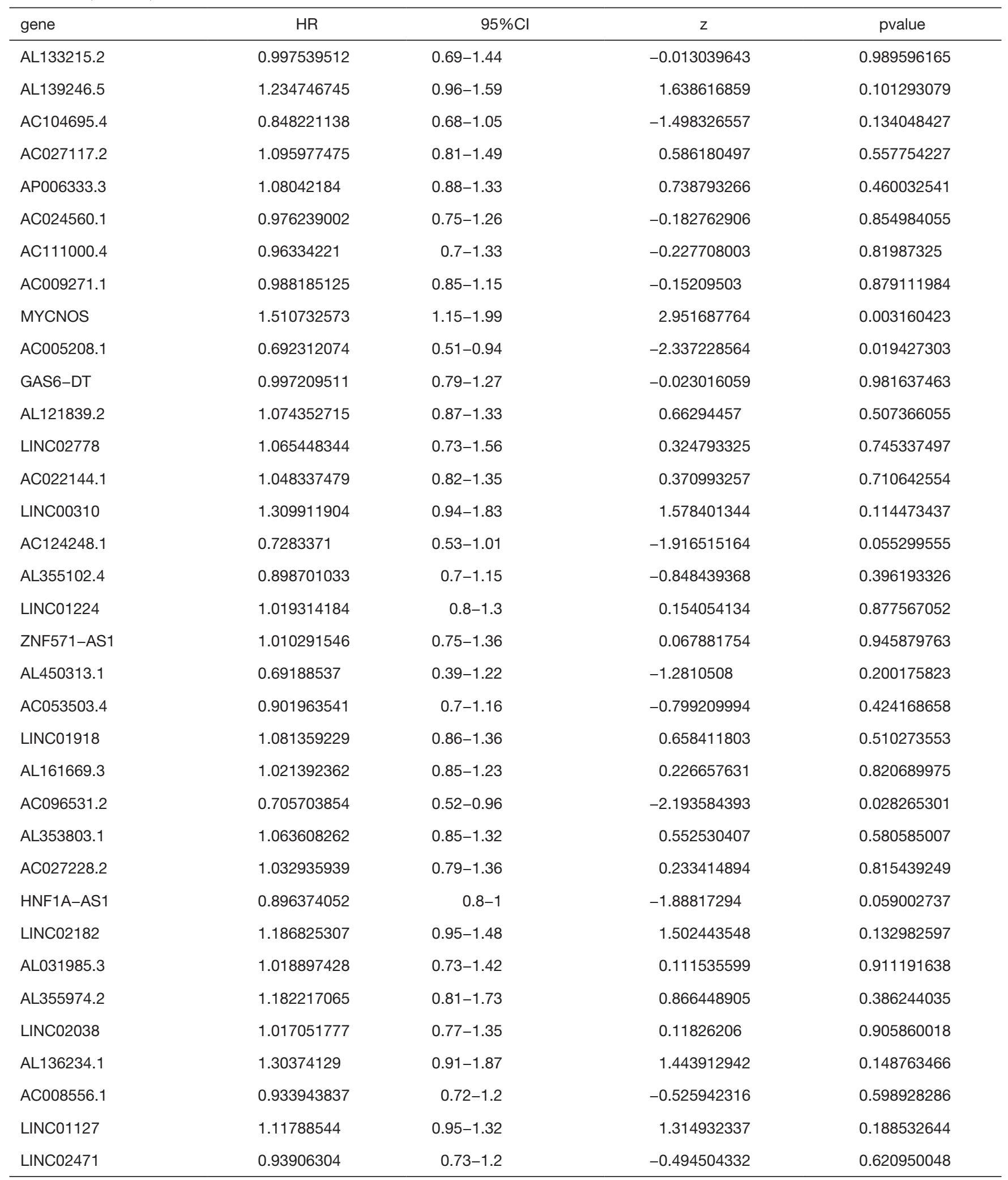

Table S2 (continued) 
Table S2 (continued)

\begin{tabular}{|c|c|c|c|c|}
\hline gene & $\mathrm{HR}$ & $95 \% \mathrm{Cl}$ & $z$ & pvalue \\
\hline AC135507.1 & 1.006336338 & $0.71-1.42$ & 0.035609124 & 0.971594033 \\
\hline AC100861.1 & 1.011034631 & $0.81-1.26$ & 0.097756614 & 0.922125559 \\
\hline SNHG1 & 1.012146123 & $0.7-1.47$ & 0.063657239 & 0.949243154 \\
\hline DSCR8 & 1.010994998 & $0.91-1.12$ & 0.205173329 & 0.837436707 \\
\hline STARD13-AS & 0.832627386 & $0.63-1.09$ & -1.313867203 & 0.188890897 \\
\hline NAV2-AS3 & 1.266869309 & $1.02-1.58$ & 2.109190481 & 0.034928143 \\
\hline AL162586.1 & 1.004384817 & $0.71-1.41$ & 0.025121577 & 0.97995799 \\
\hline SNHG6 & 0.708810352 & $0.48-1.05$ & -1.696618504 & 0.089768809 \\
\hline LINC01357 & 0.951815359 & $0.71-1.28$ & -0.330244711 & 0.741215066 \\
\hline CHL1-AS2 & 1.061935316 & $0.89-1.27$ & 0.664029102 & 0.506671685 \\
\hline AL592424.1 & 0.889874171 & $0.65-1.22$ & -0.730553299 & 0.465052046 \\
\hline SNHG28 & 1.157523856 & $0.91-1.48$ & 1.165706526 & 0.243733121 \\
\hline AL157373.2 & 1.001614002 & $0.75-1.34$ & 0.010960102 & 0.991255279 \\
\hline LHFPL3-AS2 & 0.894915731 & $0.79-1.01$ & -1.786894356 & 0.073954565 \\
\hline AL590226.1 & 1.178970425 & $0.89-1.57$ & 1.131892901 & 0.257679464 \\
\hline LINC02418 & 0.951368816 & $0.83-1.09$ & -0.699119985 & 0.48447705 \\
\hline AC008649.2 & 1.117411392 & $0.89-1.41$ & 0.949461276 & 0.342386058 \\
\hline SACS-AS1 & 0.866462944 & $0.7-1.08$ & -1.288281096 & 0.197648131 \\
\hline LINC01770 & 1.289014855 & $1.01-1.65$ & 2.035245522 & 0.041826175 \\
\hline AC093627.7 & 0.903553543 & $0.76-1.08$ & -1.110095839 & 0.26695773 \\
\hline AC116563.1 & 1.140868467 & $0.98-1.33$ & 1.685088232 & 0.09197155 \\
\hline LINC01738 & 1.060605235 & $0.89-1.27$ & 0.646838497 & 0.517736474 \\
\hline AC022613.1 & 1.084733074 & $0.81-1.46$ & 0.541866274 & 0.587910631 \\
\hline LINC01539 & 1.085846918 & $0.88-1.35$ & 0.752802572 & 0.451568561 \\
\hline AC097059.1 & 0.998557979 & $0.8-1.24$ & -0.013026364 & 0.989606759 \\
\hline AP001363.2 & 1.078448332 & $0.87-1.34$ & 0.674039924 & 0.500285932 \\
\hline
\end{tabular}

Table S2 (continued) 
Table S2 (continued)

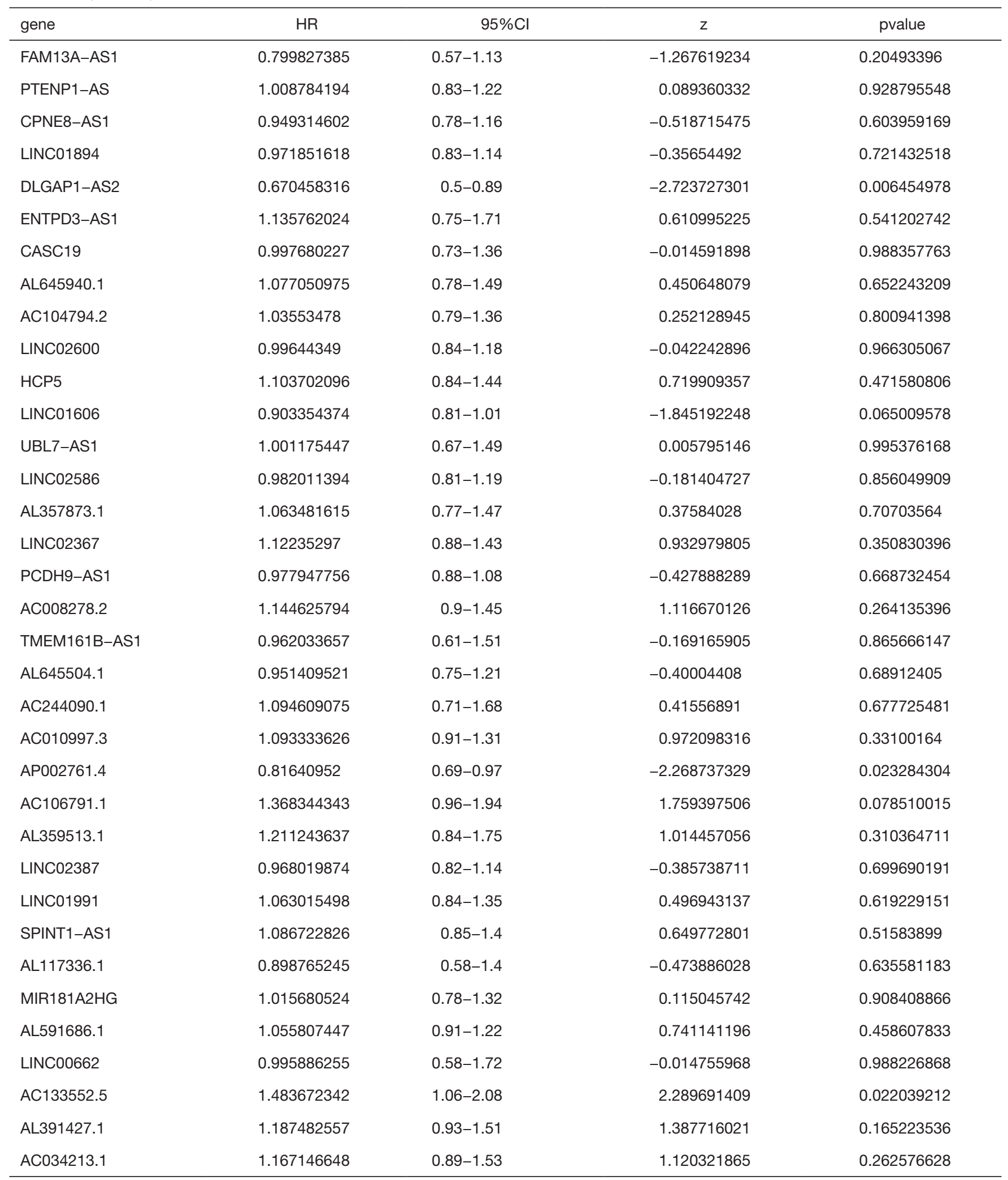

Table S2 (continued) 
Table S2 (continued)

\begin{tabular}{|c|c|c|c|c|}
\hline gene & $\mathrm{HR}$ & $95 \% \mathrm{Cl}$ & $z$ & pvalue \\
\hline AC106875.1 & 0.948383323 & $0.8-1.13$ & -0.607128044 & 0.543765941 \\
\hline BX255923.1 & 1.035217443 & $0.85-1.25$ & 0.354119439 & 0.723249375 \\
\hline LNCOG & 0.959822127 & $0.78-1.19$ & -0.380573274 & 0.703519916 \\
\hline AL359076.1 & 1.082645244 & $0.81-1.44$ & 0.540710231 & 0.588707326 \\
\hline RASGRF2-AS1 & 1.130001356 & $0.87-1.47$ & 0.90907719 & 0.363309382 \\
\hline AL139275.2 & 1.015229617 & $0.83-1.24$ & 0.145750505 & 0.88411835 \\
\hline AC021517.1 & 0.864532431 & $0.66-1.14$ & -1.046614053 & 0.295277618 \\
\hline AC004466.2 & 0.94725853 & $0.71-1.26$ & -0.368570296 & 0.712448039 \\
\hline AP001269.2 & 0.94489224 & $0.65-1.38$ & -0.294514527 & 0.768364765 \\
\hline NFIA-AS2 & 0.920053313 & $0.76-1.12$ & -0.844568912 & 0.398351573 \\
\hline GAS5 & 1.02074474 & $0.77-1.34$ & 0.145923358 & 0.883981892 \\
\hline AL121944.2 & 1.695050155 & $1.12-2.57$ & 2.478939216 & 0.013177376 \\
\hline AC011447.3 & 0.927934496 & $0.72-1.19$ & -0.589372759 & 0.555611246 \\
\hline AC093157.1 & 1.294633451 & $0.83-2.01$ & 1.145727379 & 0.251907972 \\
\hline SNHG29 & 0.825488515 & $0.58-1.17$ & -1.078291502 & 0.280903688 \\
\hline AC008035.1 & 0.929416531 & $0.68-1.28$ & -0.451419863 & 0.65168697 \\
\hline AC023983.2 & 1.020348113 & $0.77-1.36$ & 0.137498789 & 0.89063655 \\
\hline U62631.1 & 1.041205234 & $0.83-1.31$ & 0.347893924 & 0.727919849 \\
\hline SNHG5 & 0.905399646 & $0.65-1.26$ & -0.589001205 & 0.555860465 \\
\hline LINC01498 & 1.113968783 & $0.91-1.37$ & 1.024914937 & 0.305403326 \\
\hline AC003101.2 & 0.968329238 & $0.75-1.25$ & -0.248848705 & 0.803477815 \\
\hline LINC01558 & 0.871847844 & $0.73-1.05$ & -1.470436353 & 0.141443612 \\
\hline FAM78B-AS1 & 1.074491199 & $0.89-1.3$ & 0.733396725 & 0.463316496 \\
\hline AL024507.3 & 1.09236897 & $0.73-1.64$ & 0.427682957 & 0.66888196 \\
\hline LINC01485 & 1.041672569 & $0.82-1.32$ & 0.335105134 & 0.73754578 \\
\hline DLEU1 & 0.948293293 & $0.64-1.41$ & -0.263825701 & 0.79191423 \\
\hline
\end{tabular}

Table S2 (continued) 
Table S2 (continued)

\begin{tabular}{|c|c|c|c|c|}
\hline gene & $\mathrm{HR}$ & $95 \% \mathrm{Cl}$ & z & pvalue \\
\hline AC023043.4 & 0.958984277 & $0.75-1.22$ & -0.337738708 & 0.735560102 \\
\hline AC005674.2 & 1.169715306 & $0.93-1.48$ & 1.321018515 & 0.186495191 \\
\hline STARD7-AS1 & 0.99638617 & $0.68-1.46$ & -0.018679725 & 0.985096602 \\
\hline MAPKAPK5-AS1 & 0.702725281 & $0.42-1.18$ & -1.342908411 & 0.179301633 \\
\hline ZFAS1 & 0.94859326 & $0.68-1.32$ & -0.316604288 & 0.751543882 \\
\hline PLCG1-AS1 & 1.534674843 & $1.05-2.25$ & 2.189463204 & 0.02856319 \\
\hline LINC01111 & 1.032070526 & $0.85-1.26$ & 0.309398897 & 0.75701811 \\
\hline CCNT2-AS1 & 1.04843346 & $0.71-1.54$ & 0.241501888 & 0.809166153 \\
\hline NALCN-AS1 & 1.069647919 & $0.89-1.29$ & 0.715481155 & 0.474311782 \\
\hline MTUS2-AS1 & 1.062389912 & $0.88-1.28$ & 0.637759656 & 0.523630146 \\
\hline AC073046.1 & 1.044827433 & $0.88-1.24$ & 0.49313456 & 0.621917513 \\
\hline LINC00861 & 1.048248888 & $0.81-1.36$ & 0.357227883 & 0.720921213 \\
\hline AC023043.1 & 0.966278972 & $0.78-1.19$ & -0.317638805 & 0.750758936 \\
\hline AC068492.1 & 1.148300853 & $0.9-1.46$ & 1.122202788 & 0.261776228 \\
\hline AC005746.2 & 0.939512785 & $0.75-1.17$ & -0.547902722 & 0.583758699 \\
\hline AL035461.3 & 1.133990522 & $0.82-1.57$ & 0.750347559 & 0.453045406 \\
\hline TM4SF1-AS1 & 0.933680712 & $0.72-1.21$ & -0.525541856 & 0.599206564 \\
\hline AC129507.1 & 0.825402951 & $0.68-1$ & -2.010555867 & 0.04437239 \\
\hline TRAF3IP2-AS1 & 1.018863665 & $0.68-1.52$ & 0.09162078 & 0.926999341 \\
\hline AC097478.1 & 0.940075198 & $0.79-1.11$ & -0.715324017 & 0.474408851 \\
\hline AC234772.2 & 1.246357782 & $1.02-1.52$ & 2.149084618 & 0.031627693 \\
\hline PCAT19 & 1.025514371 & $0.8-1.32$ & 0.194492155 & 0.845790538 \\
\hline KAZN-AS1 & 1.162953972 & $0.87-1.56$ & 1.004909973 & 0.314940202 \\
\hline AC090403.1 & 1.139624265 & $0.93-1.4$ & 1.251918002 & 0.210599744 \\
\hline AC007780.1 & 1.01791835 & $0.75-1.37$ & 0.116064311 & 0.907601573 \\
\hline AL391832.2 & 1.066864399 & $0.85-1.34$ & 0.553637949 & 0.579826649 \\
\hline
\end{tabular}

Table S2 (continued) 
Table S2 (continued)

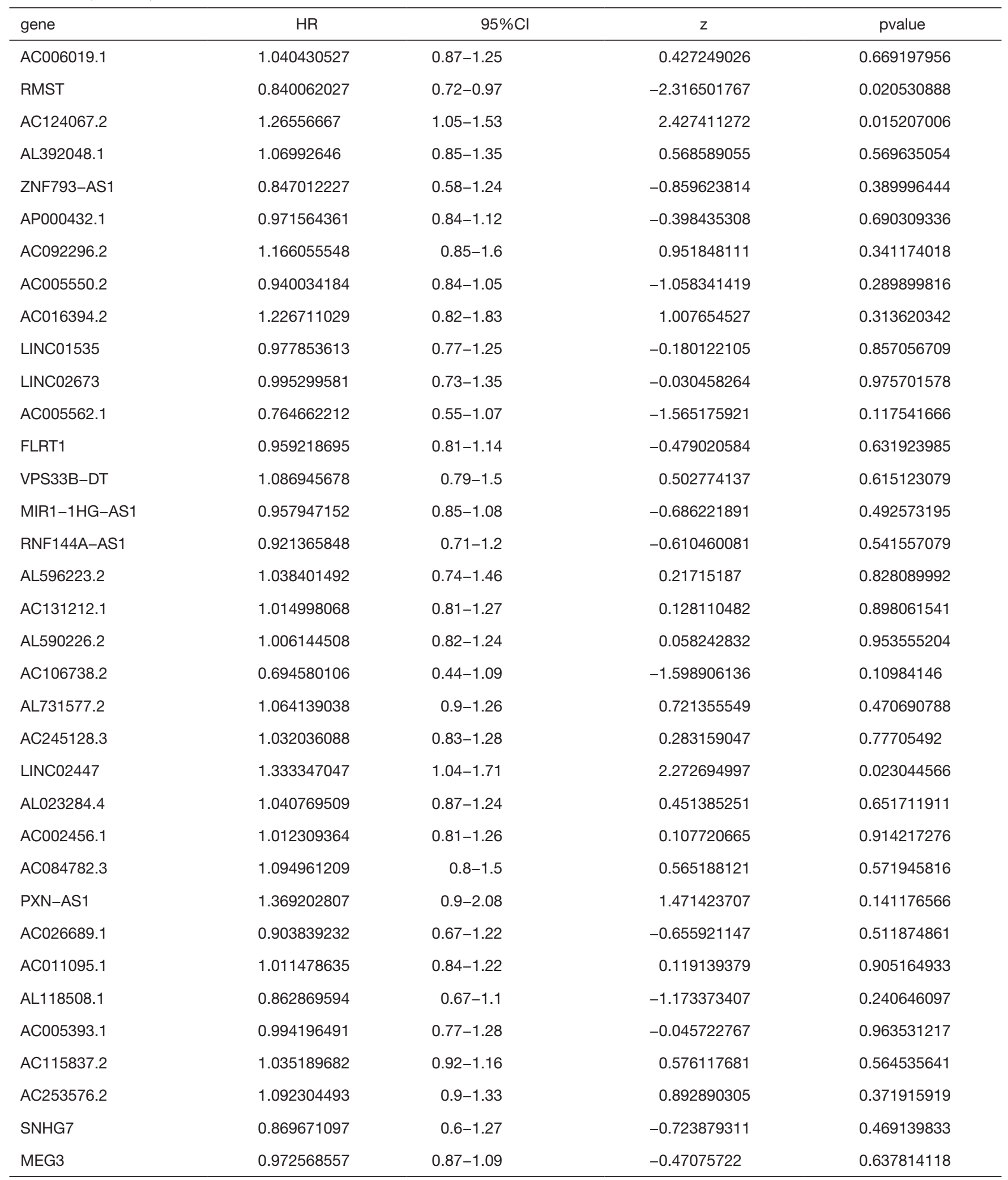

Table S2 (continued) 
Table S2 (continued)

\begin{tabular}{|c|c|c|c|c|}
\hline gene & $\mathrm{HR}$ & $95 \% \mathrm{Cl}$ & z & pvalue \\
\hline AC055822.1 & 0.969914627 & $0.72-1.3$ & -0.202918049 & 0.839199086 \\
\hline JPX & 0.841037341 & $0.52-1.36$ & -0.702991019 & 0.482061346 \\
\hline PRKAR1B-AS2 & 0.991805375 & $0.87-1.13$ & -0.122644542 & 0.902388581 \\
\hline AC037198.1 & 1.01962393 & $0.81-1.28$ & 0.164679033 & 0.869196621 \\
\hline MEG8 & 0.944214811 & $0.82-1.09$ & -0.772987885 & 0.43952955 \\
\hline AC012085.2 & 0.821377666 & $0.64-1.05$ & -1.570536704 & 0.116290302 \\
\hline AF038458.2 & 0.863126 & $0.65-1.14$ & -1.036844929 & 0.299808133 \\
\hline AC107953.2 & 0.916471993 & $0.66-1.28$ & -0.509992068 & 0.610057018 \\
\hline AL358473.2 & 0.946490427 & $0.8-1.12$ & -0.626571115 & 0.530940408 \\
\hline AC145285.6 & 1.116202864 & $0.81-1.55$ & 0.659556755 & 0.509538313 \\
\hline AC012636.1 & 0.872435215 & $0.72-1.06$ & -1.351482434 & 0.176540943 \\
\hline PIK3CD-AS2 & 1.111220548 & $0.8-1.54$ & 0.637740111 & 0.523642871 \\
\hline LINC01697 & 0.92682289 & $0.8-1.07$ & -1.040733848 & 0.297999089 \\
\hline AC023403.1 & 0.948087262 & $0.77-1.17$ & -0.491341558 & 0.623184891 \\
\hline AC011468.1 & 0.778469316 & $0.54-1.12$ & -1.357597886 & 0.174591314 \\
\hline LINC02041 & 1.033918759 & $0.9-1.19$ & 0.460034488 & 0.645491466 \\
\hline AL080317.1 & 0.876383261 & $0.59-1.31$ & -0.64395371 & 0.519605451 \\
\hline AF279873.3 & 1.017460216 & $0.84-1.24$ & 0.174443626 & 0.861516832 \\
\hline AC092354.1 & 0.985948247 & $0.71-1.37$ & -0.084985882 & 0.932272615 \\
\hline DNAH10OS & 1.095647602 & $0.87-1.37$ & 0.789394938 & 0.429881213 \\
\hline SMG7-AS1 & 1.20470336 & $0.94-1.55$ & 1.448320077 & 0.147527555 \\
\hline KIRREL3-AS3 & 0.811120556 & $0.66-0.99$ & -2.031136083 & 0.042241192 \\
\hline AC025811.1 & 0.93158073 & $0.8-1.09$ & -0.890437572 & 0.373230976 \\
\hline AC018529.2 & 0.94616987 & $0.76-1.18$ & -0.485223849 & 0.627517566 \\
\hline AL109804.1 & 0.770718129 & $0.53-1.11$ & -1.394735816 & 0.16309552 \\
\hline PCED1B-AS1 & 0.981822939 & $0.76-1.27$ & -0.138673042 & 0.889708521 \\
\hline
\end{tabular}

Table S2 (continued) 
Table S2 (continued)

\begin{tabular}{|c|c|c|c|c|}
\hline gene & $\mathrm{HR}$ & $95 \% \mathrm{Cl}$ & z & pvalue \\
\hline AC110772.2 & 0.913412929 & $0.78-1.08$ & -1.083178893 & 0.278729027 \\
\hline AC134312.5 & 0.977458627 & $0.79-1.2$ & -0.214356946 & 0.830268713 \\
\hline SNHG3 & 1.342231007 & $0.94-1.91$ & 1.625392928 & 0.104078862 \\
\hline AL390728.6 & 1.025959374 & $0.7-1.5$ & 0.131337048 & 0.895508685 \\
\hline AC039056.2 & 0.930466815 & $0.74-1.17$ & -0.607128429 & 0.543765685 \\
\hline LINC01675 & 1.112659954 & $0.85-1.45$ & 0.780352416 & 0.435183468 \\
\hline AL355336.2 & 0.878553897 & $0.68-1.13$ & -0.992995247 & 0.320712271 \\
\hline AL391261.2 & 1.017748316 & $0.82-1.27$ & 0.158010003 & 0.874448916 \\
\hline AC023669.2 & 1.183382145 & $0.86-1.63$ & 1.0311697 & 0.302461247 \\
\hline LINC02674 & 1.065443133 & $0.8-1.43$ & 0.42521096 & 0.670682895 \\
\hline AC010425.1 & 0.886255006 & $0.74-1.06$ & -1.349588427 & 0.177148038 \\
\hline AP001993.1 & 0.847261127 & $0.68-1.06$ & -1.441197176 & 0.149528986 \\
\hline AC123912.4 & 0.882439842 & $0.76-1.03$ & -1.579798728 & 0.114152967 \\
\hline AL357054.4 & 0.99579142 & $0.79-1.25$ & -0.036510406 & 0.970875382 \\
\hline AC243585.1 & 0.907433795 & $0.66-1.24$ & -0.608781311 & 0.542669403 \\
\hline AC010967.1 & 1.118954039 & $0.97-1.29$ & 1.552849076 & 0.120459195 \\
\hline Z82214.2 & 0.997199017 & $0.81-1.22$ & -0.027029006 & 0.978436599 \\
\hline AC127029.2 & 0.878185968 & $0.7-1.11$ & -1.099812219 & 0.271413947 \\
\hline LINC02677 & 0.953341613 & $0.7-1.3$ & -0.300393826 & 0.763876773 \\
\hline MAP3K5-AS1 & 1.081024879 & $0.85-1.37$ & 0.637024222 & 0.524109066 \\
\hline AL021807.1 & 0.971228413 & $0.76-1.24$ & -0.233012898 & 0.81575139 \\
\hline AC005224.3 & 0.923839209 & $0.82-1.05$ & -1.240252142 & 0.214882148 \\
\hline AC011500.3 & 1.15893732 & $0.93-1.45$ & 1.286938809 & 0.198115617 \\
\hline LINC00944 & 1.001098788 & $0.89-1.13$ & 0.017727376 & 0.985856341 \\
\hline AC073525.1 & 0.907817948 & $0.76-1.09$ & -1.0382816 & 0.299138973 \\
\hline AC022079.1 & 1.166118838 & $0.86-1.58$ & 0.986361082 & 0.323955956 \\
\hline
\end{tabular}

Table S2 (continued) 
Table S2 (continued)

\begin{tabular}{|c|c|c|c|c|}
\hline gene & HR & $95 \% \mathrm{Cl}$ & z & pvalue \\
\hline AC022509.3 & 1.027984819 & $0.86-1.23$ & 0.297370272 & 0.766183837 \\
\hline LAMP5-AS1 & 0.865429136 & $0.73-1.02$ & -1.68831296 & 0.091351169 \\
\hline AL596087.3 & 1.045459389 & $0.85-1.28$ & 0.433140023 & 0.664913048 \\
\hline AC092813.2 & 1.043568273 & $0.86-1.27$ & 0.429950771 & 0.667231452 \\
\hline ZBTB20-AS1 & 0.761155487 & $0.61-0.96$ & -2.334187942 & 0.019585883 \\
\hline AC096631.1 & 0.921928873 & $0.73-1.17$ & -0.665062817 & 0.506010316 \\
\hline AC016876.3 & 0.978915953 & $0.71-1.36$ & -0.128370105 & 0.897856088 \\
\hline AC063944.3 & 0.877126114 & $0.67-1.15$ & -0.946888653 & 0.343695521 \\
\hline AC129492.1 & 1.052250789 & $0.87-1.28$ & 0.514962997 & 0.606578878 \\
\hline LURAP1L-AS1 & 1.069696074 & $0.89-1.29$ & 0.71304992 & 0.475814867 \\
\hline AL121827.1 & 0.947843212 & $0.8-1.12$ & -0.639465887 & 0.522519899 \\
\hline AC087623.1 & 0.788493004 & $0.6-1.04$ & -1.669435159 & 0.095031171 \\
\hline AC100814.2 & 0.771792617 & $0.56-1.06$ & -1.599837175 & 0.109634709 \\
\hline Z68871.1 & 1.04034562 & $0.82-1.32$ & 0.32689734 & 0.743745533 \\
\hline AL139021.2 & 1.009355478 & $0.69-1.48$ & 0.047749623 & 0.961915786 \\
\hline MIR133A1HG & 0.837520034 & $0.67-1.05$ & -1.550283982 & 0.12107337 \\
\hline AC009159.2 & 0.998452156 & $0.83-1.21$ & -0.016060041 & 0.987186492 \\
\hline LINC00882 & 0.870712006 & $0.66-1.15$ & -0.978154558 & 0.327997888 \\
\hline AC012511.1 & 0.932799872 & $0.73-1.19$ & -0.55310588 & 0.580190911 \\
\hline FAM181A-AS1 & 0.930763749 & $0.78-1.11$ & -0.796336739 & 0.425836337 \\
\hline AC097478.2 & 0.951758382 & $0.8-1.14$ & -0.549242728 & 0.582838886 \\
\hline AC005288.1 & 0.839268839 & $0.65-1.09$ & -1.335808968 & 0.181611721 \\
\hline AL356056.1 & 0.925839052 & $0.72-1.18$ & -0.61596329 & 0.537918749 \\
\hline LINC01063 & 1.239044621 & $0.9-1.71$ & 1.315064253 & 0.188488309 \\
\hline AF121898.1 & 0.918297561 & $0.76-1.11$ & -0.892320295 & 0.372221279 \\
\hline AC018953.1 & 1.00612598 & $0.86-1.17$ & 0.078450447 & 0.937469746 \\
\hline
\end{tabular}

Table S2 (continued) 
Table S2 (continued)

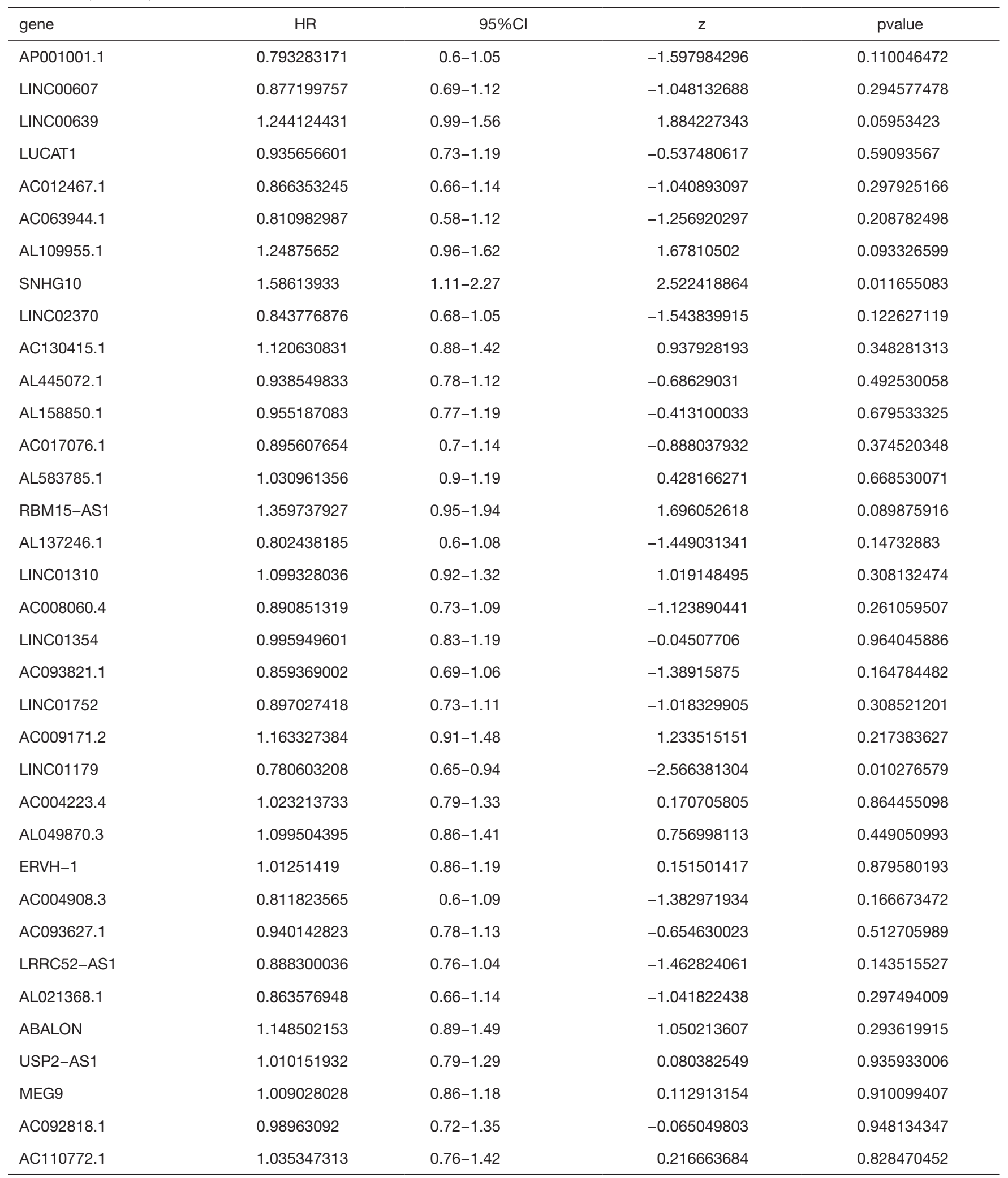

Table S2 (continued) 
Table S2 (continued)

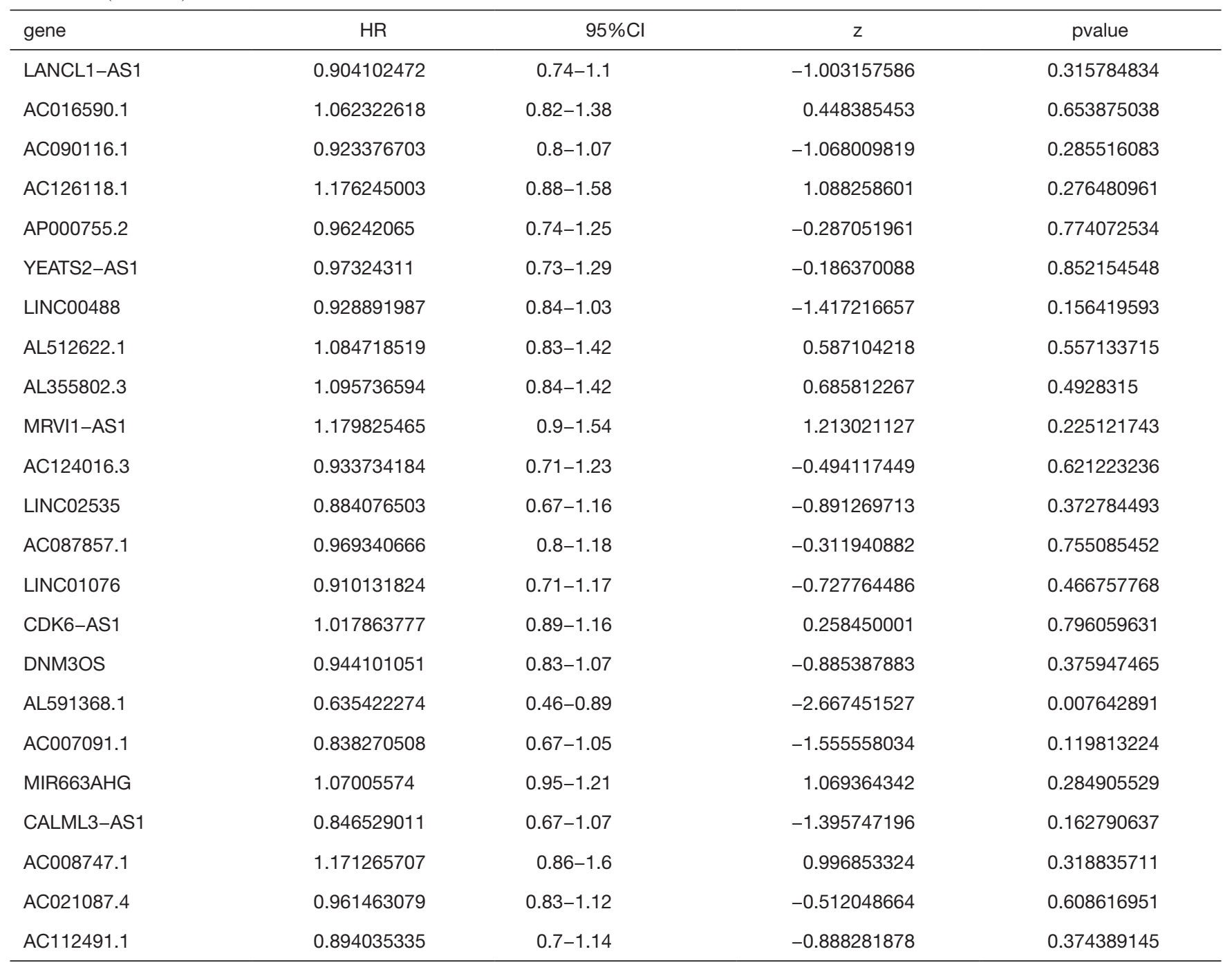


Table S3 Information of 12-lncRNA signature.

\begin{tabular}{lcccc}
\hline ID & coef & HR & Z-score & P value \\
\hline AC007406.1 & 0.3218 & 1.3797 & 1.737 & 0.0824 \\
AC005208.1 & -0.2534 & 0.7762 & -1.406 & 0.1598 \\
LINC01770 & 0.3987 & 1.4899 & 2.471 & 0.0135 \\
DLGAP1-AS2 & -0.5637 & 0.5691 & -2.911 & 0.0036 \\
AP002761.4 & -0.2012 & 0.8178 & -1.723 & 0.0849 \\
STPG3-AS1 & 0.3081 & 1.3609 & 2.104 & 0.0354 \\
AC129507.1 & -0.1872 & 0.8293 & -1.772 & 0.0764 \\
AC234772.2 & 0.2002 & 1.2216 & 1.556 & 0.1197 \\
LINC02447 & 0.3068 & 1.3591 & 1.813 & 0.0699 \\
AC009570.1 & 0.44 & 1.5528 & 2.094 & 0.0363 \\
ZBTB20-AS1 & -0.3948 & 0.6738 & -2.418 & 0.0156 \\
LINC01179 & -0.2656 & 0.7667 & -2.319 & 0.0204 \\
\hline
\end{tabular}

Table S4 Univariate Cox regression analysis of clinical characteristics and the signature-based risk score

\begin{tabular}{lcccc}
\hline ID & HR & HR.95L & HR.95H & pvalue \\
\hline age & 0.983615242 & 0.889051437 & 1.088237308 & 0.748713173 \\
gender & 1.751037265 & 1.003521121 & 3.055373165 & 0.048570908 \\
race & 1.214454169 & 0.654454756 & 2.253630089 & 0.537926351 \\
stage & 1.536873711 & 1.114254481 & 2.119785778 & 0.00880939 \\
histologic & 1.118336134 & 0.622792782 & 2.008173095 & 0.708057547 \\
riskScore & 1.095083879 & 1.06638845 & 1.124551472 & $2.0216 \mathrm{E}-11$ \\
\hline
\end{tabular}

Table S5 Multivariate Cox regression analysis of clinical characteristics and the signature-based risk score

\begin{tabular}{lcccc}
\hline ID & HR & HR.95L & HR.95H & pvalue \\
\hline age & 0.987944604 & 0.879397266 & 1.109890352 & 0.838164267 \\
gender & 1.801824255 & 0.974675179 & 3.330925746 & 0.060361629 \\
race & 1.181834081 & 0.609020138 & 2.293408228 & 0.621371753 \\
stage & 1.56060184 & 1.09353305 & 2.227164603 & 0.014178586 \\
histologic & 1.498859176 & 0.800614987 & 2.806066419 & 0.205899698 \\
riskScore & 1.08560966 & 1.053582128 & 1.118610787 & $7.60727 \mathrm{E}-08$ \\
\hline
\end{tabular}

